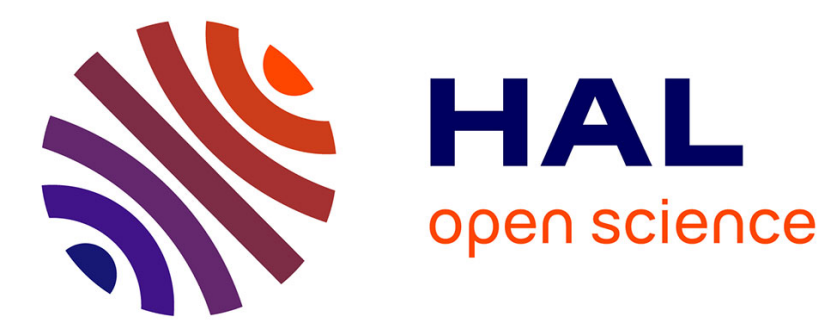

\title{
A semiparametric extension of the stochastic block model for longitudinal networks
}

Catherine Matias, Tabea Rebafka, Fanny Villers

\section{To cite this version:}

Catherine Matias, Tabea Rebafka, Fanny Villers. A semiparametric extension of the stochastic block model for longitudinal networks: Semiparametric estimation in PPSBM. Biometrika, 2018, 105 (3), pp.665-680. 10.1093/biomet/asy016 . hal-01245867v3

\section{HAL Id: hal-01245867 \\ https://hal.science/hal-01245867v3}

Submitted on 21 Jul 2017

HAL is a multi-disciplinary open access archive for the deposit and dissemination of scientific research documents, whether they are published or not. The documents may come from teaching and research institutions in France or abroad, or from public or private research centers.
L'archive ouverte pluridisciplinaire HAL, est destinée au dépôt et à la diffusion de documents scientifiques de niveau recherche, publiés ou non, émanant des établissements d'enseignement et de recherche français ou étrangers, des laboratoires publics ou privés. 


\title{
A semiparametric extension of the stochastic block model for longitudinal networks
}

\author{
Catherine Matias, Tabea Rebafka and Fanny Villers \\ Sorbonne Universités, Université Pierre et Marie Curie, Université Paris Diderot, Centre \\ National de la Recherche Scientifique, Laboratoire de Probabilités et Modèles Aléatoires, \\ 4 place Jussieu, 75252 PARIS Cedex 05, FRANCE. \\ \{catherine.matias, tabea.rebafka, fanny.villers\}@upmc.fr
}

\begin{abstract}
To model recurrent interaction events in continuous time, an extension of the stochastic block model is proposed where every individual belongs to a latent group and interactions between two individuals follow a conditional inhomogeneous Poisson process with intensity driven by the individuals' latent groups. The model is shown to be identifiable and its estimation is based on a semiparametric variational expectation-maximization algorithm. Two versions of the method are developed, using either a nonparametric histogram approach (with an adaptive choice of the partition size) or kernel intensity estimators. The number of latent groups can be selected by an integrated classification likelihood criterion. Finally, we demonstrate the performance of our procedure on synthetic experiments, analyse two datasets to illustrate the utility of our approach and comment on competing methods.
\end{abstract}

Keywords: dynamic interactions; expectation-maximization algorithm; integrated classification likelihood; link streams; longitudinal network; semiparametric model; variational approximation; temporal network.

\section{Introduction}

The past few years have seen a large increase in the interest for modelling dynamic interactions between individuals. Continuous-time information on interactions is often available, as e.g. email exchanges between employees in a company (Klimt \& Yang, 2004) or face-to-face contacts between individuals measured by sensors (Stehlé et al., 2011), but most models use discrete time. Commonly, data are aggregated on predefined time intervals to obtain a sequence of snapshots of random graphs. Besides the loss of information induced by aggregation, the specific choice of the time intervals has a direct impact on the results, most often overlooked. Thus, developing continuous-time models - either called longitudinal networks, interaction event data, link streams or temporal networks - is an important research issue.

Statistical methods for longitudinal networks form a huge corpus, especially in social sciences and we are not exhaustive here, see Holme (2015) for a more complete review. It is natural to model temporal event data by stochastic point processes. An important line of research involves continuous-time Markov processes with seminal works on dyad-independent models (Wasserman, 
1980b a) or the so-called stochastic actor oriented models (e.g. Snijders \& van Duijn, 1997; Snijders et al., 2010). In these works interactions last during some time. In contrast, here we focus on instantaneous interactions identified with time points. Furthermore, we are concerned with modelling dependencies between interactions of pairs of individuals.

The analysis of event data is an old area in statistics (see e.g. Andersen et al., 1993). Generally, the number of interactions of all pairs $(i, j)$ of individuals up to time $t$ are modelled by a multivariate counting process $N(t)=\left(N_{i, j}(t)\right)_{(i, j)}$. Butts (2008) considers time-stamped interactions marked by a label representing a behavioural event. His model is an instance of Cox's multiplicative hazard model with time-dependent covariates and constant baseline function. In the same vein, Vu et al. (2011) propose a regression-based modelling of the intensity of non recurrent interaction events. They consider two different frameworks: Cox's multiplicative and Aalen's additive hazard rates. Perry \& Wolfe (2013) propose another variant of Cox's multiplicative intensity model for recurrent interaction events where the baseline function is specific to each individual. In the above mentioned works a set of statistics is chosen by the user that potentially modulate the interactions. As in any regression framework, the choice of these statistics raises some issues: increasing their number may lead to a high-dimensional problem, and interpretation of the results might be blurred by their possible correlations.

The approaches by Butts, Vu et al., Perry \& Wolfe and others are based on conditional Poisson processes characterized by random intensities, also known as doubly stochastic Poisson processes or Cox processes. An instance of the conditional Poisson process is the Hawkes process, which is a collection of point processes with some background rate, where each event adds a nonnegative impulse to the intensity of all other processes. Introducing reciprocating Hawkes to parameterize edges Blundell et al. (2012) extend the Infinite Relational Model making all events co-dependent over time. Cho et al. (2014) develop a model for spatial-temporal networks with missing information, based on Hawkes processes for temporal dynamics combined with a Gaussian mixture for the spatial dynamics. Similarly, Linderman \& Adams (2014) combine temporal Hawkes processes with latent distance models for implicit networks that are not directly observed. We also mention the existence of models associating point processes with single nodes rather than pairs, see e.g. Fox et al. (2016) and the references therein.

Clustering individuals based on interaction data is a well-established technique to take into account the intrinsic heterogeneity and summarize information. For discrete-time sequences of graphs, recent approaches propose generalizations of the stochastic block model to a dynamic context (Yang et al., 2011; Xu \& Hero, 2014; Corneli et al., 2016; Matias \& Miele, to appear). Stochastic block models posit that all individuals belong to one out of finitely many groups and given these groups all pairs of interactions are independent. We stress that stochastic block models induce more general clusterings than community detection algorithms. Indeed, clusters are not necessarily characterized by intense within-group interaction and low interaction frequency towards other groups. Another attempt to use stochastic block models for interaction events appears in DuBois et al. (2013) generalizing the approach of Butts (2008) by adding discrete latent variables on the individuals.

We introduce a semiparametric stochastic block model for recurrent interaction events in continuous time, to which we refer as the Poisson process stochastic block model. Interactions are modelled by conditional inhomogeneous Poisson processes, whose intensities only depend on the latent groups of the interacting individuals. In contrast to many other works, we do not rely on a parametric model where intensities are modulated by predefined network statistics, but intensities are modelled and estimated in a nonparametric way. The model parameters are shown to 
be identifiable. Our estimation and clustering approach is a semiparametric version of the variational expectation-maximization algorithm, where the maximization step is replaced by nonparametric estimators of the intensities. Semiparametric generalizations of the classical expectationmaximization algorithm have been proposed in many different contexts; see e.g. Böhning (1995); Bordes et al. (2007); Robin et al. (2007) for semiparametric mixtures or Dannemann (2012) for a semiparametric hidden Markov model. However, we are not aware of other attempts to incorporate nonparametric estimates in a variational approximation algorithm. We propose two different estimations of the nonparametric part of the model: a histogram approach using Reynaud-Bouret (2006) where the partition size is adaptively chosen and a kernel estimator based on Ramlau-Hansen (1983). With the histogram approach, an integrated classification likelihood criterion is proposed to select the number of latent groups. Synthetic experiments and the analysis of two datasets illustrate the strengths and weaknesses of our approach. The $\mathrm{R}$ code is available in the $\mathrm{R}$ package ppstom.

\section{A semiparametric Poisson process stochastic block model}

\subsection{Model}

We consider the pairwise interactions of $n$ individuals during some time interval $[0, T]$. For notational convenience we restrict our attention to directed interactions without self-interactions. The undirected case and self-interactions are handled similarly. The set of all pairs of individuals (i.e. the set of all possible dyads in the graph) is denoted

$$
\mathcal{R}=\{(i, j): i, j=1, \ldots, n ; i \neq j\},
$$

whose cardinality is $r=n(n-1)$. We observe the interactions during the time interval $[0, T]$, that is

$$
\mathcal{O}=\left\{\left(t_{m}, i_{m}, j_{m}\right), m=1, \ldots, M\right\},
$$

where $\left(t_{m}, i_{m}, j_{m}\right) \in[0, T] \times \mathcal{R}$ corresponds to the event that a (directed) interaction from the $i_{m}$ th individual to the $j_{m}$ th individual occurs at time $t_{m}$. The total (random) number of events is $M$. We assume that $0<t_{1}<\cdots<t_{M}<T$, i.e. there is at most one event at a time.

Every individual is assumed to belong to one out of $Q$ groups and the relation between two individuals, that is the way they interact with another, is driven by their group membership. Let $Z_{1}, \ldots, Z_{n}$ be independent and identically distributed (latent) random variables taking values in $\{1, \ldots, Q\}$ with non zero probabilities $\operatorname{pr}\left(Z_{1}=q\right)=\pi_{q}(q=1, \ldots, Q)$. For the moment, $Q$ is considered to be fixed and known. When no confusion occurs, we also use the notation $Z_{i}=$ $\left(Z^{i, 1}, \ldots, Z^{i, Q}\right)$ with $Z^{i, q} \in\{0,1\}$ such that $Z_{i}$ has multinomial distribution $\mathcal{M}(1, \pi)$ with $\pi=$ $\left(\pi_{1}, \ldots, \pi_{Q}\right)$.

For every pair $(i, j) \in \mathcal{R}$ the interactions of individual $i$ to $j$ conditional on the latent groups $Z_{1}, \ldots, Z_{n}$ are modelled by a conditional inhomogeneous Poisson process $N_{i, j}(\cdot)$ on $[0, T]$ with intensity depending only on $Z_{i}$ and $Z_{j}$. For $q, l=1, \ldots, Q$ and $(i, j) \in \mathcal{R}$ the conditional intensity of $N_{i, j}(\cdot)$ given that $Z_{i}=q$ and $Z_{j}=l$ is $\alpha^{(q, l)}(\cdot)$ with corresponding cumulative intensity

$$
A^{(q, l)}(t)=\int_{0}^{t} \alpha^{(q, l)}(u) d u, \quad(0 \leq t \leq T) .
$$

The set of observations $\mathcal{O}$ is a realization of the multivariate counting process $\left\{N_{i, j}(\cdot)\right\}_{(i, j) \in \mathcal{R}}$ with conditional intensity process $\left\{\alpha^{\left(Z_{i}, Z_{j}\right)}(\cdot)\right\}_{(i, j) \in \mathcal{R}}$. The process $N_{i, j}$ is not a Poisson process, 
but a counting process with intensity $\sum_{q=1}^{Q} \sum_{l=1}^{Q} \pi_{q} \pi_{l} \alpha^{(q, l)}$. We denote $\theta=(\pi, \alpha)$ the infinitedimensional parameter of our model and $\mathrm{pr}_{\theta}$ the Poisson process stochastic block model distribution

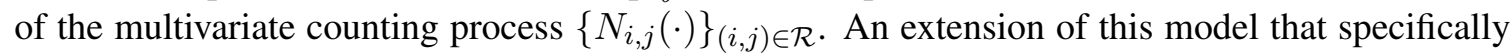
accounts for sparse interactions processes is given in the Supplementary Material.

\subsection{Identifiability}

Identifiability of the parameter $\theta$ corresponds to injectivity of the mapping $\theta \mapsto \operatorname{pr}_{\theta}$ and may be obtained at best up to label switching, as defined below. We denote $\mathfrak{S}_{Q}$ the set of permutations of $\{1, \ldots, Q\}$.

Definition 1 (Identifiability up to label switching). The parameter $\theta=(\pi, \alpha)$ of a Poisson process stochastic block model is identifiable on $[0, T]$ up to label switching if for all $\theta$ and $\tilde{\theta}$ such that $p r_{\theta}=p r_{\tilde{\theta}}$ there exists a permutation $\sigma \in \mathfrak{S}_{Q}$ such that

$$
\pi_{q}=\tilde{\pi}_{\sigma(q)}, \quad \alpha^{(q, l)}=\tilde{\alpha}^{(\sigma(q), \sigma(l))} \text { almost everywhere on }[0, T], \quad(q, l=1, \ldots, Q) .
$$

Identifiability (up to label switching) is ensured in the very general setting where the intensities $\alpha^{(q, l)}$ are not equal almost everywhere, that is, they may be identical on at most subsets of $[0, T]$.

Assumption 1. In the directed setup (resp. undirected), the set of intensities $\left\{\alpha^{(q, l)}\right\}_{q, l=1, \ldots, Q}$ contains exactly $Q^{2}$ (resp. $Q(Q+1) / 2$ ) distinct functions.

Proposition 1. Under Assumption 1 , the parameter $\theta=(\pi, \alpha)$ is identifiable on $[0, T]$ up to label switching from the Poisson process stochastic block model distribution $p_{\theta}$, as soon as $n \geq 3$.

Assumption 1 is similar to the hypothesis from Theorem 12 in Allman et al. (2011) that to our knowledge is the only identifiability result for weighted stochastic block models. The question whether the necessary condition that any two rows (or any two columns) of the parameter matrix $\alpha$ are distinct is a sufficient condition for identifiability is not yet answered even in the simpler binary case. In the binary stochastic block model, the results in Allman et al. (2009, 2011) establish generic identifiability, which means identifiability except on a subset of parameters with Lebesgue measure zero, without specifying this subset. For the directed and binary stochastic block model, Celisse et al. (2012) establish identifiability under the assumption that the product vector $\alpha \pi$ (or $\pi^{\top} \alpha$ ) has distinct coordinates. This condition is slightly stronger than requiring any two rows of the parameter matrix to be distinct. Another identifiability result appears in Bickel et al. (2011) for some specific block models. These last two approaches are dedicated to the binary setup and cannot be generalized to the continuous case.

Proposition 1 does not cover the undirected affiliation case, where only two intensities $\alpha^{\text {in }}$ and $\alpha^{\text {out }}$ are considered such that $\alpha^{(q, q)}=\alpha^{\text {in }}$ and $\alpha^{(q, l)}=\alpha^{\text {out }}(q, l=1, \ldots, Q ; q \neq l)$.

Proposition 2. If the intensities $\alpha^{\text {in }}$ and $\alpha^{\text {out }}$ are distinct functions on $[0, T]$, then both $\alpha^{\text {in }}$ and $\alpha^{\text {out }}$ are identifiable on $[0, T]$ from the undirected affiliation Poisson process stochastic block model distribution $p_{\theta}$ when $n \geq 3$. Moreover, for $n \geq \max \{Q, 3\}$, the proportions $\pi_{1}, \ldots, \pi_{Q}$ are also identifiable up to a permutation. 


\subsection{Additional notation}

We introduce some quantities that are relevant in the following. Denote

$$
\begin{aligned}
Y_{\mathcal{Z}}^{(q, l)} & =\sum_{(i, j) \in \mathcal{R}} Z^{i, q} Z^{j, l}, \quad(q, l=1, \ldots, Q), \\
N_{\mathcal{Z}}^{(q, l)} & =\sum_{(i, j) \in \mathcal{R}} Z^{i, q} Z^{j, l} N_{i, j}, \quad(q, l=1, \ldots, Q), \\
Z_{m}^{(q, l)} & =Z^{i_{m}, q} Z^{j_{m}, l}, \quad(q, l=1, \ldots, Q ; m=1, \ldots, M) .
\end{aligned}
$$

These are the (latent) number of dyads $(i, j) \in \mathcal{R}$ with latent groups $(q, l)$, the (latent) counting process of interactions between individuals in groups $(q, l)$ and the (latent) binary indicator of observation $\left(t_{m}, i_{m}, j_{m}\right)$ belonging to groups $(q, l)$, respectively. As these quantities are unobserved, our work relies on proxies based on approximations of the latent group variables $Z^{i, q}$. Denote

$$
\mathcal{T}=\left\{\tau=\left(\tau^{i, q}\right)_{i=1, \ldots, n, q=1, \ldots, Q}: \tau^{i, q} \geq 0, \sum_{q=1}^{Q} \tau^{i, q}=1, \quad(i=1, \ldots, n ; q=1, \ldots, Q)\right\} .
$$

While the variables $Z^{i, q}$ are indicators, their counterparts $\tau^{i, q}$ are weights representing the probability that node $i$ belongs to group $q$. Now, for every $\tau \in \mathcal{T}$, replacing all latent variables $Z^{i, q}$ in (1)-(3) by $\tau^{i, q}$, we define $Y^{(q, l)}, N^{(q, l)}$ and $\tau_{m}^{(q, l)}$ which are estimators of $Y_{\mathcal{Z}}^{(q, l)}, N_{\mathcal{Z}}^{(q, l)}$ and $Z_{m}^{(q, l)}$, respectively.

\section{Semiparametric estimation procedure}

\subsection{A variational semiparametric expectation-maximization algorithm}

The complete-data likelihood of observations $\mathcal{O}$ and latent variables $\mathcal{Z}=\left(Z_{1}, \ldots, Z_{n}\right)$ is

$$
\mathcal{L}(\mathcal{O}, \mathcal{Z} \mid \theta)=\exp \left\{-\sum_{(i, j) \in \mathcal{R}} A^{\left(Z_{i}, Z_{j}\right)}(T)\right\} \prod_{m=1}^{M} \alpha^{\left(Z_{i_{m}}, Z_{j_{m}}\right)}\left(t_{m}\right) \prod_{i=1}^{n} \prod_{q=1}^{Q} \pi_{q}^{Z^{i, q}} .
$$

The likelihood of the observed data $\mathcal{L}(\mathcal{O} \mid \theta)$ is obtained by summing the above over the set of all possible configurations of the latent variables $\mathcal{Z}$. This set is huge and thus the likelihood $\mathcal{L}(\mathcal{O} \mid \theta)$ is intractable for direct maximization. Hence, an expectation-maximization algorithm (Dempster et al., 1977) is used, which is an iterative procedure especially adapted to cope with latent variables. It consists of an E-step and an M-step that are iterated until convergence. In our model two issues arise. First, as already observed for the standard stochastic block model (Daudin et al., 2008), the Estep requires the computation of the conditional distribution of $\mathcal{Z}$ given the observations $\mathcal{O}$, which is not tractable. Therefore, we use a variational approximation (Jordan et al., 1999) of the latent variables' conditional distribution to perform the E-step. We refer to Matias \& Robin (2014) for a presentation and a discussion on the variational approximation in stochastic block models. Second, part of our parameter is infinite dimensional so that the M-step is partly replaced by a nonparametric estimation procedure, giving rise to a semiparametric algorithm. 


\subsection{Variational E-step}

The standard E-step consists in computing the expectation of the complete-data log-likelihood given the observations at some current parameter value $\theta$. This requires the knowledge of the conditional latent variables distribution $\operatorname{pr}_{\theta}(\mathcal{Z} \mid \mathcal{O})$, which is not tractable because the $Z_{i}$ 's are not conditionally independent. We thus perform a variational approximation of $\operatorname{pr}_{\theta}(\mathcal{Z} \mid \mathcal{O})$ by a simpler distribution. Using the class of parameters $\mathcal{T}$ defined by (4) we consider the family of factorized distributions on $\mathcal{Z}$ given $\mathcal{O}$ defined as

$$
\operatorname{pr}_{\tau}\left\{\mathcal{Z}=\left(q_{1}, \ldots, q_{n}\right) \mid \mathcal{O}\right\}=\prod_{i=1}^{n} \operatorname{pr}_{\tau}\left(Z_{i}=q_{i} \mid \mathcal{O}\right)=\prod_{i=1}^{n} \tau^{i, q_{i}}, \quad\left(q_{1}, \ldots, q_{n}\right) \in\{1, \ldots, Q\}^{n}
$$

with corresponding expectation $E_{\tau}(\cdot \mid \mathcal{O})$. Denoting $\operatorname{KL}\{\cdot \| \cdot\}$ the Kullback-Leibler divergence, we search for the parameter $\hat{\tau} \in \mathcal{T}$ that yields the best approximation $\operatorname{pr}_{\tau}(\cdot \mid \mathcal{O})$ of $\operatorname{pr}_{\theta}(\cdot \mid \mathcal{O})$ through

$$
\hat{\tau}=\underset{\tau \in \mathcal{T}}{\operatorname{Argmin}} \operatorname{KL}\left\{\operatorname{pr}_{\tau}(\cdot \mid \mathcal{O}) \| \operatorname{pr}_{\theta}(\cdot \mid \mathcal{O})\right\} .
$$

Let $\mathcal{H}(\cdot)$ be the entropy of a distribution. It can be seen that $\hat{\tau}$ maximizes with respect to $\tau$ the quantity

$$
\begin{aligned}
J(\theta, \tau) & =E_{\tau}\{\log \mathcal{L}(\mathcal{O}, \mathcal{Z} \mid \theta) \mid \mathcal{O}\}+\mathcal{H}\left\{\operatorname{pr}_{\tau}(\cdot \mid \mathcal{O})\right\} \\
& =-\sum_{q=1}^{Q} \sum_{l=1}^{Q} Y^{(q, l)} A^{(q, l)}(T)+\sum_{q=1}^{Q} \sum_{l=1}^{Q} \sum_{m=1}^{M} \tau_{m}^{(q, l)} \log \left\{\alpha^{(q, l)}\left(t_{m}\right)\right\}+\sum_{i=1}^{n} \sum_{q=1}^{Q} \tau^{i, q} \log \left(\frac{\pi_{q}}{\tau^{i, q}}\right) .
\end{aligned}
$$

The solution $\hat{\tau}$ satisfies a fixed point equation, which in practice is found by successively updating the variational parameters $\tau^{i, q}$ via the following Equation (6) until convergence.

Proposition 3. The solution $\hat{\tau}$ to the minimization problem (5) satisfies the fixed point equation

$$
\hat{\tau}^{i, q} \propto \pi_{q} \exp \left\{D_{i q}(\hat{\tau}, \alpha)\right\}, \quad(i=1, \ldots, n ; q=1, \ldots, Q),
$$

where $\propto$ means 'proportional to', $\mathbb{1}_{A}$ denotes the indicator function of set $A$ and

$$
\begin{aligned}
D_{i q}(\tau, \alpha)= & -\sum_{l=1}^{Q} \sum_{\substack{j=1 \\
j \neq i}}^{n} \tau^{j, l}\left\{A^{(q, l)}(T)+A^{(l, q)}(T)\right\} \\
& +\sum_{l=1}^{Q} \sum_{m=1}^{M}\left[\mathbb{1}_{\left\{i_{m}=i\right\}} \tau^{j_{m}, l} \log \left\{\alpha^{(q, l)}\left(t_{m}\right)\right\}+\mathbb{1}_{\left\{j_{m}=i\right\}} \tau^{i_{m}, l} \log \left\{\alpha^{(l, q)}\left(t_{m}\right)\right\}\right] .
\end{aligned}
$$

\subsection{Nonparametric M-step: general description}

In a parametric context the M-step consists in the maximization of $J(\theta, \tau)$ with respect to $\theta=(\pi, \alpha)$. Considering only the finite-dimensional part $\pi$ of the parameter, the maximizer $\hat{\pi}$ is

$$
\hat{\pi}_{q}=\frac{\sum_{i=1}^{n} \tau^{i, q}}{\sum_{q=1}^{Q} \sum_{i=1}^{n} \tau^{i, q}}=\frac{1}{n} \sum_{i=1}^{n} \tau^{i, q}, \quad(q=1, \ldots, Q) .
$$


Concerning the infinite-dimensional parameter $\alpha$, we replace the maximization of $J(\pi, \alpha, \tau)$ with respect to $\alpha$ by a nonparametric estimation step. If the process $N_{\mathcal{Z}}^{(q, l)}$ defined by (2) was observed, the estimation of $\alpha^{(q, l)}$ would be straightforward. Now the criterion $J$ depends on $\alpha$ through the quantity $E_{\tau}(\log \mathcal{L}(\mathcal{O}, \mathcal{Z} \mid \theta) \mid \mathcal{O})$, that corresponds to the log-likelihood in a setup where we observe the weighted cumulative process $N^{(q, l)}$ (see Section 2.3). Intensities may be easily estimated in this direct observation setup. We develop two different approaches for updating $\alpha$ : a histogram and a kernel method.

\subsection{Histogram-based M-step}

In this part the intensities $\alpha^{(q, l)}$ are estimated by piecewise constant functions and we propose a data-driven choice of the partition of the time interval $[0, T]$. The procedure is based on a leastsquares penalized criterion following the work of Reynaud-Bouret (2006). Here $(q, l)$ is fixed and for reasons of computational efficiency we focus on regular dyadic partitions that form embedded sets of partitions.

For some given $d_{\max }$, consider all regular dyadic partitions of $[0, T]$ into $2^{d}$ intervals

$$
\mathcal{E}_{d}=\left\{E_{k}=\left[(k-1) \frac{T}{2^{d}} ; k \frac{T}{2^{d}}\right): k=1, \ldots, 2^{d}\right\}, \quad\left(d=0, \ldots, d_{\max }\right) .
$$

For any interval $E$ included in $[0, T]$, the estimated mean number of observed interactions $\left(t_{m}, i_{m}, j_{m}\right)$ between individuals with latent groups $(q, l)$ and occurring in $E$ is

$$
N^{(q, l)}(E)=\int_{E} d N^{(q, l)}(s)=\sum_{m=1}^{M} \tau^{i_{m}, q} \tau^{j_{m}, l_{1}} \mathbb{1}_{E}\left(t_{m}\right) .
$$

The total number of dyads $r$ is an upper bound for $Y^{(q, l)}$, so we define a least-squares contrast (relatively to the counting process $\left.N^{(q, l)}\right)$ for all $f \in \mathbb{L}^{2}([0, T], d t)$ by

$$
\gamma^{(q, l)}(f)=-\frac{2}{r} \int_{0}^{T} f(t) d N^{(q, l)}(t)+\frac{Y^{(q, l)}}{r} \int_{0}^{T} f^{2}(t) d t .
$$

The projection estimator of $\alpha^{(q, l)}$ on the set $S_{d}$ of piecewise constant functions on $\mathcal{E}_{d}$ is

$$
\hat{\alpha}_{d}^{(q, l)}=\underset{f \in S_{d}}{\operatorname{argmin}} \gamma^{(q, l)}(f)=\frac{1}{Y^{(q, l)}} \sum_{E \in \mathcal{E}_{d}} \frac{N^{(q, l)}(E)}{|E|} \mathbb{1}_{E}(\cdot),
$$

where $|E|$ is the length of interval $E$, here equal to $T 2^{-d}$. Now, adaptive estimation consists in choosing the best estimator among the collection $\left\{\hat{\alpha}_{d}^{(q, l)}: d=0, \ldots, d_{\max }\right\}$. So we introduce an estimator of the partition through $\hat{d}^{(q, l)}$ minimizing a penalized least-squares criterion of the form

$$
\operatorname{crit}^{(q, l)}(d)=\gamma^{(q, l)}\left(\hat{\alpha}_{d}^{(q, l)}\right)+\operatorname{pen}^{(q, l)}(d),
$$

for some penalty function $\operatorname{pen}^{(q, l)}:\left\{0, \ldots, d_{\max }\right\} \rightarrow \mathbb{R}^{+}$that penalizes large partitions. We choose the penalty function as

$$
\operatorname{pen}^{(q, l)}(d)=\frac{2^{d+1}}{r} C \quad \text { with } \quad C=\frac{2^{d_{\max }}}{T Y^{(q, l)}} \sup _{E \in \mathcal{E}_{d_{\max }}} N^{(q, l)}(E) .
$$


Finally, the penalized least-squares criterion simplifies to

$$
\hat{d}^{(q, l)}=\underset{d=0, \ldots, d_{\max }}{\operatorname{Argmin}} 2^{d}\left\{-\sum_{E \in \mathcal{E}_{d}} N^{(q, l)}(E)^{2}+2^{d_{\max }+1} \sup _{E^{\prime} \in \mathcal{E}_{d_{\max }}} N^{(q, l)}\left(E^{\prime}\right)\right\} .
$$

The selected partition size $\hat{d}=\hat{d}^{(q, l)}$ may be specific to the groups $(q, l)$. Finally, the adaptive estimator of intensity $\alpha^{(q, l)}$ is

$$
\hat{\alpha}_{\text {hist }}^{(q, l)}(t)=\hat{\alpha}_{\hat{d}}^{(q, l)}(t)=\frac{2^{\hat{d}}}{T Y^{(q, l)}} \sum_{E \in \mathcal{E}_{\hat{d}}} N^{(q, l)}(E) \mathbb{1}_{E}(t),
$$

where $0 \leq t \leq T ; q, l=1, \ldots, Q$. Reynaud-Bouret (2006) develops her approach in the Aalen multiplicative intensity model, which is slightly different from our context. Moreover, our setup does not satisfy the assumptions of Theorem 1 in that reference as the number of jumps of the processes $N_{i, j}$ is not bounded by a known value (we have recurrent events). Nevertheless, in our simulations this procedure correctly estimates the intensities $\alpha^{(q, l)}$ (see Section 4 ). We refer to Baraud \& Birgé (2009) for a theoretical study of an adaptive nonparametric estimation of the intensity of a Poisson process. Reynaud-Bouret (2006) also studies other penalized least-squares estimators (for e.g. using Fourier bases), which might be used here similarly.

\subsection{Kernel-based M-step}

Kernel methods are suited to estimate smooth functions. If the variational parameters $\tau^{i, q}$ are good approximations of the latent variables $Z^{i, q}$, then the intensity of process $N^{(q, l)}$ (Section 2.3 is approximately $Y^{(q, l)} \alpha^{(q, l)}$, where $Y^{(q, l)}$ is the variational mean number of dyads with latent groups $(q, l)$. Following Ramlau-Hansen (1983) and considering a nonnegative kernel function $K$ with support within $[-1,1]$ together with some bandwidth $b>0$, the intensity $\alpha^{(q, l)}$ is estimated by

$$
\hat{\alpha}_{\mathrm{ker}}^{(q, l)}(t)=\frac{1}{b Y^{(q, l)}} \int_{0}^{T} K\left(\frac{t-u}{b}\right) d N^{(q, l)}(u)=\frac{1}{b Y^{(q, l)}} \sum_{m=1}^{M} \tau_{m}^{(q, l)} K\left(\frac{t-t_{m}}{b}\right),
$$

if $Y^{(q, l)}>0$ and $\hat{\alpha}_{\mathrm{ker}}^{(q, l)}(t)=0$ otherwise, where $\tau_{m}^{(q, l)}$ is defined in Section 2.3 .

The bandwidth $b$ could be chosen adaptively from the data following the procedure proposed by Grégoire (1993). Kernel methods are not always suited to infer a function on a bounded interval as boundary effects may deteriorate their quality. However, it is out of the scope of this work to investigate refinements of this kind.

\subsection{Algorithm's full description}

The implementation of the algorithm raises two issues: convergence and initialization. As our algorithm is an iterative procedure, one has to test for convergence. A stopping criterion can be defined based on criterion $J(\theta, \tau)$. Concerning initialization the algorithm is run several times with different starting values, which are chosen by some k-means method combined with perturbation techniques (see the Supplementary Material for details). Algorithm 1 provides a full description of the procedure. 


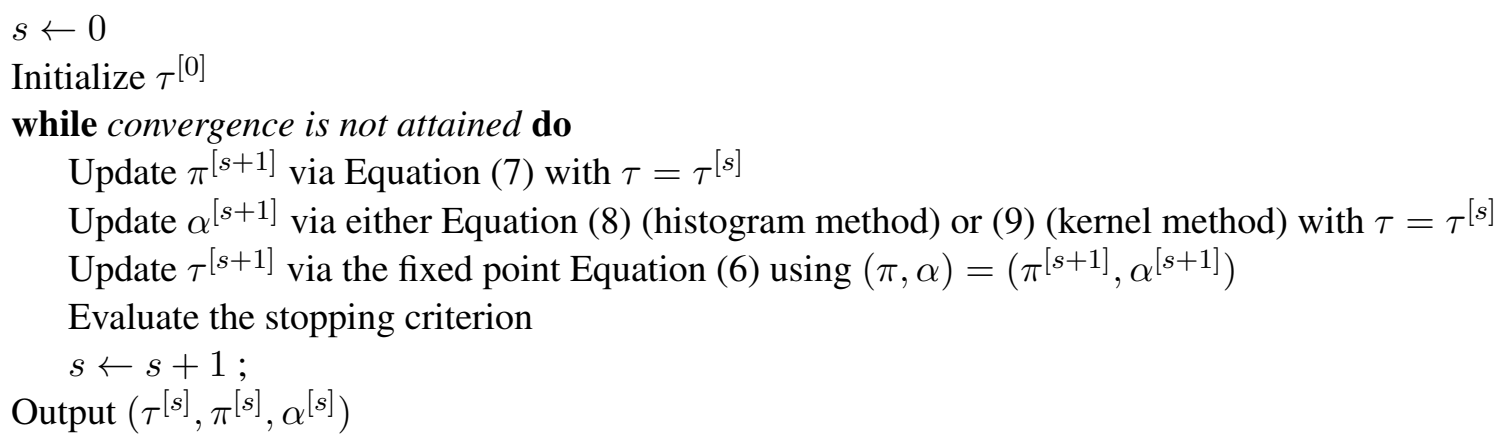

Algorithm 1: Semiparametric variational expectation-maximization algorithm

\subsection{Model selection with respect to $Q$}

We propose an integrated classification likelihood criterion that performs data-driven model selection for the number of groups $Q$. Roughly, this criterion is based on the complete-data variational log-likelihood penalized by the number of parameters. It has been introduced in the mixture context in Biernacki et al. (2000) and adapted to the stochastic block model in Daudin et al. (2008). The issue here is that our model contains a nonparametric part, so that the parameter is infinite dimensional. However in the case of histogram estimators, once the partition is selected, there is only a finite number of parameters to estimate. This number can be used to build our integrated classification likelihood criterion.

For any $Q$ let $\hat{\theta}(Q)$ be the estimated parameter and $\hat{\tau}(Q)$ the corresponding group probabilities obtained by our algorithm run with $Q$ groups. The parameter $\hat{\theta}(Q)$ has two components: the first one $\hat{\pi}(Q)$ is a vector of dimension $Q-1$, while the second has dimension $\sum_{q, l=1, \ldots, Q} \exp \left(\hat{d}^{(q, l)} \log 2\right)$. In the adaptation of the integrated classification likelihood criterion to the stochastic block model these components are treated differently: the first one, that concerns the $n$ individuals is penalized by a $\log (n) / 2$ term, while the second one concerning the dyads is penalized by a $\log (r) / 2$ term. We refer to Daudin et al. (2008) for more details. In our case the integrated classification likelihood criterion is

$$
\operatorname{ICL}(Q)=\log \mathbb{P}_{\hat{\theta}(Q)}\{\mathcal{O}, \hat{\tau}(Q)\}-\frac{1}{2}(Q-1) \log n-\frac{1}{2} \log r \sum_{q=1}^{Q} \sum_{l=1}^{Q} 2^{\hat{d}^{(q, l)}} .
$$

After fixing an upper bound $Q_{\max }$ we select the number of groups

$$
\hat{Q}=\underset{Q=1, \ldots, Q_{\max }}{\operatorname{Argmax}} \operatorname{ICL}(Q) .
$$

\section{Synthetic experiments}

We generate data using the undirected Poisson process stochastic block model in the following two scenarios.

1. To evaluate the classification performance, we consider the affiliation model with $Q=2$ groups, equal probabilities $\pi_{q}=1 / 2$ and a number of individuals $n$ in $\{10,30\}$. The intensities are sinusoids with varying shifting parameter $\varphi$ set to $\alpha^{\text {in }}(\cdot)=10\{1+\sin (2 \pi \cdot)\}$ and 

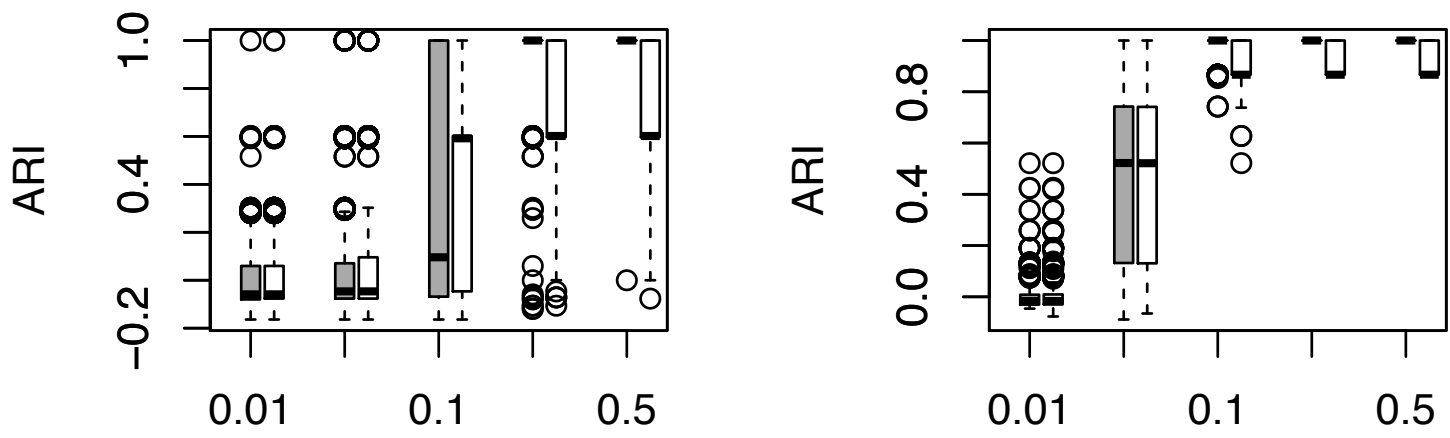

Figure 1: Boxplots of the adjusted rand index in synthetic experiments from Scenario 1 for histogram (gray) and kernel (white) estimators. The $x$-axis shows different values of $\varphi$ in $\{0.01,0.05,0.1,0.2,0.5\}$. Left panel: $n=10$, right panel: $n=30$.

$\alpha^{\text {out }}(\cdot)=10[1+\sin \{2 \pi(\cdot+\varphi)]$ with $\varphi \in\{0.01,0.05,0.1,0.2,0.5\}$. Clustering is more difficult for small values of $\varphi$.

2. To evaluate the intensity estimators, we choose $Q=3$ groups with equal probabilities $\pi_{q}=$ $1 / 3$ and six intensity functions that have different shapes and amplitudes (see continuous curves in Figure 2). The number of individuals $n$ varies in $\{20,50\}$.

For every parameter setting, our algorithm is applied to 1000 simulated datasets. The histogram estimator is used with regular dyadic partitions and $d_{\max }=3$, while the kernel estimator uses the Epanechnikov kernel.

To assess the clustering performance, we use the adjusted rand index (Hubert \& Arabie, 1985) that evaluates the agreement between the estimated and the true latent structure. For two classifications that are identical (up to label switching), this index equals 1, otherwise the adjusted rand index is smaller than 1 and negative values are possible. Figure 1 shows the boxplots of the adjusted rand index obtained with the histogram and the kernel versions of our method in Scenario 1. For small values of the shifting parameter $(\varphi \in\{0.01,0.05\})$, the intensities are so close that classification is very difficult, especially when $n=10$ is small. The classification improves when the shift between the intensities and/or the number of observations increase, achieving (almost) perfect classification for larger values of $\varphi$ and/or $n$.

Concerning the recovery of the intensities in Scenario 2, we define the risk measuring the distance between the true intensity $\alpha^{(q, l)}$ and its estimate $\hat{\alpha}^{(q, l)}$ as

$$
\operatorname{RISK}(q, l)=\left\|\hat{\alpha}^{(q, l)}-\alpha^{(q, l)}\right\|_{2}=\left[\int_{0}^{T}\left\{\hat{\alpha}^{(q, l)}(t)-\alpha^{(q, l)}(t)\right\}^{2} d t\right]^{1 / 2} .
$$

Table 1 reports mean values and standard deviations of the risk when $n=50$ (The results for $n=20$ are given in the Supplementary Material). Our histogram and kernel estimators are compared to their 'oracle' equivalents obtained using the knowledge of the true groups. The table also reports the mean number of events with latent groups $(q, l)$. As expected, when the true intensity is piecewiseconstant, the histogram version of our method outperforms the kernel estimator. Conversely, when 
Table 1: Mean number of events and risks with standard deviations (sd) in Scenario 2 with $n=50$. Histogram (Hist) and kernel (Ker) estimators are compared with their oracle counterparts (Or.Hist, Or.Ker). All values associated with the risks are multiplied by 100 .

$\begin{array}{lccccc}\text { Groups }(q, l) & \text { Nb.events } & \text { Hist (sd) } & \text { Or.Hist (sd) } & \text { Ker (sd) } & \text { Or.Ker (sd) } \\ & & & & & \\ (1,1) & 543 & 31(32) & 20(18) & 81(51) & 63(12) \\ (1,2) & 949 & 44(17) & 81(4) & 172(57) & 156(7) \\ (1,3) & 545 & 46(16) & 38(6) & 53(88) & 21(6) \\ (2,2) & 212 & 69(10) & 70(9) & 50(56) & 35(11) \\ (2,3) & 844 & 187(6) & 185(2) & 125(56) & 106(11) \\ (3,3) & 298 & 83(13) & 81(13) & 64(53) & 43(12)\end{array}$

the true intensity is smooth, the kernel estimator is more appropriate to recover the shape of the intensity. Both estimators exhibit good performances with respect to the oracles versions.

Finally, we use Scenario 2 to illustrate the performance of the integrated classification likelihood criterion to select the number $Q$ of latent groups from the data. For each of the 1000 simulated datasets, the maximizer $\hat{Q}$ of the integrated classification likelihood criterion defined in $(10)$ with $Q_{\max }=10$ is computed. For $n=20$ the correct number of groups is recovered in $74 \%$ of the cases (remaining cases select values in $\{2,4\}$ ). Moreover, for datasets where the criterion does not select the correct number $Q$, the adjusted rand index of the classification obtained with 3 groups is rather low indicating that the algorithm has failed in the classification task and probably only a local maximum of the criterion $J$ has been found.

For $n=50$ our procedure selects the correct number of groups in $99.9 \%$ of the cases.

\section{Datasets}

\subsection{London cycles dataset}

We use the cycle hire usage data from the bike sharing system of the city of London from 2012 to 2015 (Transport for London, 2016). We focus on two randomly chosen weekdays, 1st (day1) and 2nd (day2) February 2012. Data consist in pairs of stations associated with a single hiring/journey (departure station, ending station) and corresponding time stamp (hire time with second precision). The datasets have been pre-processed to remove journeys that either correspond to loops, last less than 1 minute or more than 3 hours or do not have an ending station (lost or stolen bikes). The datasets contain $n_{1}=415$ and $n_{2}=417$ stations on day 1 and day 2 with $M_{1}=17,631$ and $M_{2}=16,333$ hire events respectively. With more than $17 \times 10^{4}$ oriented pairs of stations the number of processes $N_{i, j}$ is huge, but only a very small fraction - around 7\% - of these processes are non null (i.e. contain at least one hiring event between these stations). Indeed bike sharing systems are mostly used for short trips and stations far from another are unlikely to be connected. Data correspond to origin/destination flows and are analysed in a directed setup with the histogram version of our algorithm on a regular dyadic partitions with maximum size $32\left(d_{\max }=5\right)$.

The integrated classification likelihood criterion achieves its maximum with $\hat{Q}=6$ latent groups for both datasets. Geographic locations of the bike stations and the clusters are represented on a city map (thanks to the OpenStreetMap project), see Figure 3 for day 1 . Clusters for day 2 


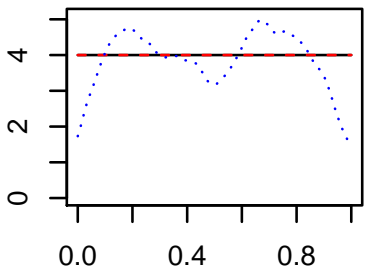

$(1,1)$

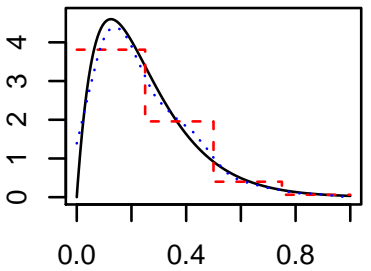

$(2,2)$

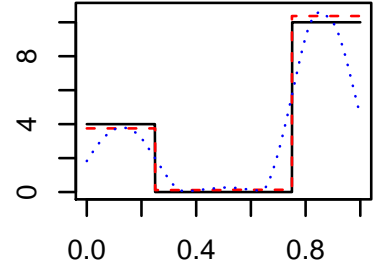

$(1,2)$

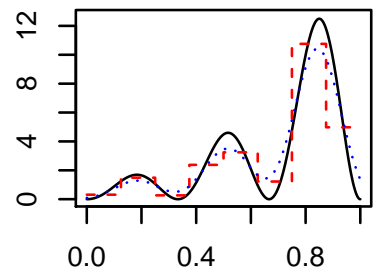

$(2,3)$

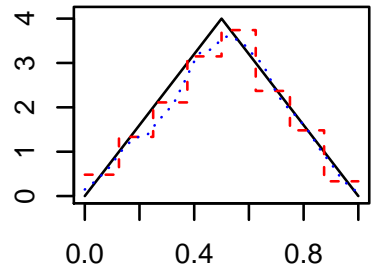

$(1,3)$

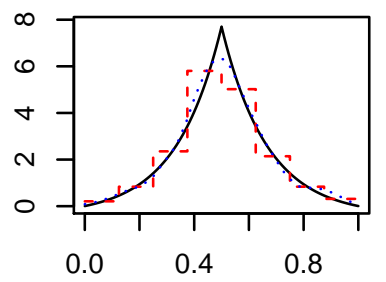

$(3,3)$

Figure 2: Intensities in synthetic experiments from Scenario 2 with $n=50$. Each panel displays for a pair of groups $(q, l)$ (given in the $x$-label) with $q, l=$ $1, \ldots, 3 ; q \leq l$ the true intensity (continuous), histogram (dashed) and kernel (dotted) estimates for one simulated dataset picked at random.

are similar thus we focus on day 1 . Our procedure globally recovers geographic clusters, as interacting stations are expected to be geographically close. A closer look at the clusters then reveals more information. While all but one clusters contain between 17 and 202 stations, cluster number 4 (dark blue crosses in Figure 3) consists of only two bike stations. They are located at Kings Cross railway and Waterloo railway stations and are among the stations with the highest activities (for both departures and arrivals) in comparison to all other stations. These stations appear to be 'outgoing' stations in the morning with much more departures than arrivals around 8a.m. and 'incoming' stations at the end of the day, with more arrivals than departures between $5 \mathrm{p} . \mathrm{m}$ and $7 \mathrm{p} . \mathrm{m}$. The bike stations close to the two other main railway stations in London (Victoria and Liverpool Street stations) do not exhibit the same pattern and are clustered differently despite having a large number of hiring events. This cluster is thus created from the similarity of the temporal profiles of these two stations rather than their total amount of interactions. Moreover it mostly interacts with cluster number 5 shown in light blue diamond in Figure 3 which roughly corresponds to the City of London neighbourhood. It is thus characterized by stations used by people living in the suburbs and working in the city center.

We compare our method with the discrete-time approach developed in Matias \& Miele (to appear) where individuals are allowed to change groups during time. We applied the corresponding dynsbm $\mathrm{R}$ package on the London bikes dataset aggregated into $T=24$ snapshots of one hour 


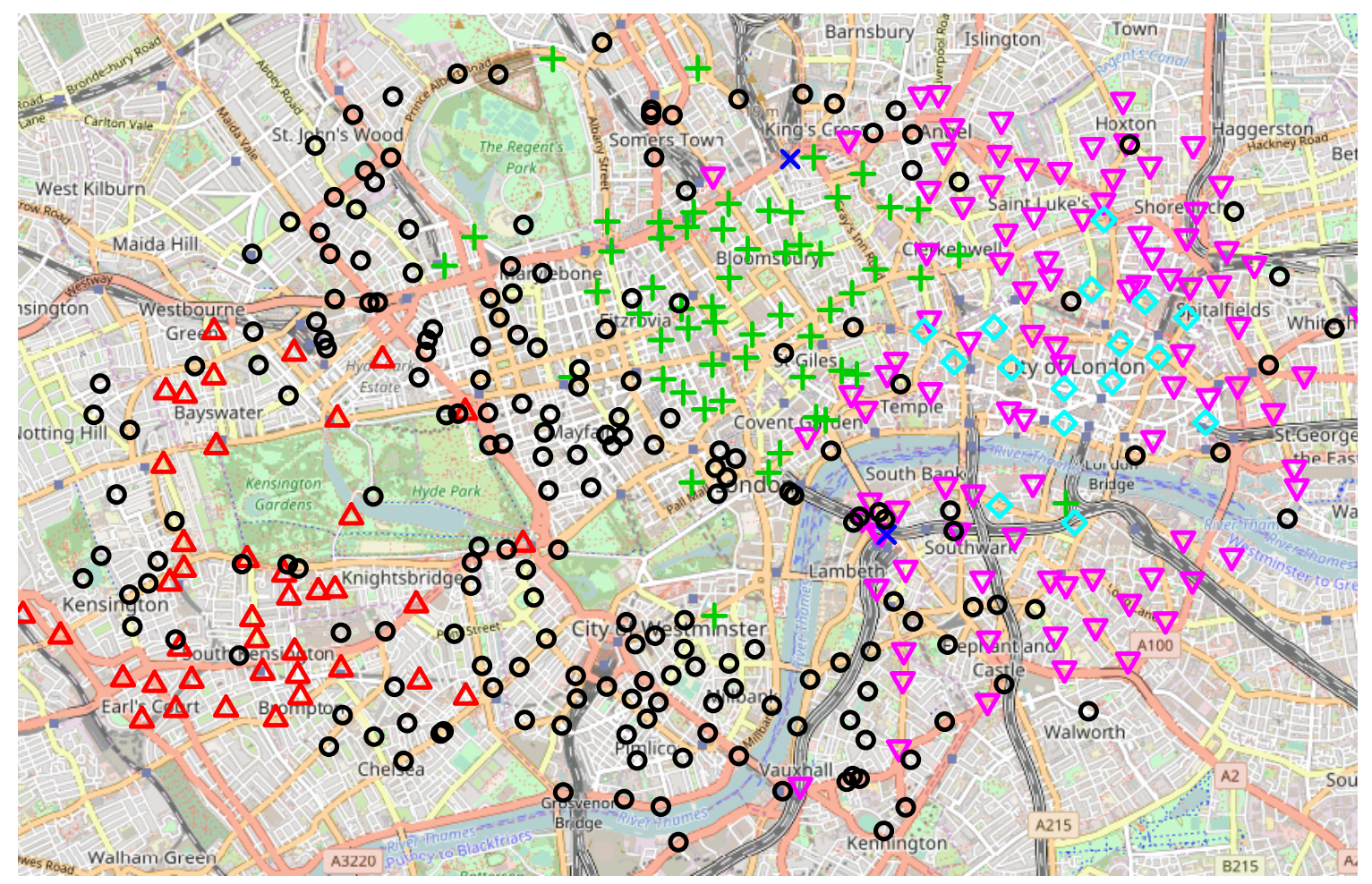

Figure 3: London bike sharing system: Geographic positions of the stations and clustering into six clusters (represented by different colors and symbols) for day 1 .

long, but no similar result came out of this: their model selection criterion chooses 4 groups. The clusters with $Q=4$ groups in fact drop to only 1 nonempty group between midnight to 3am, 2 groups between $3 \mathrm{am}$ and $7 \mathrm{am}$ and 3 groups between $10 \mathrm{pm}$ and midnight. Globally there is one very large group (containing from 168 to all stations, with mean number 285), one medium size group (from 0 to 148 stations, mean value 93) and 2 small groups (from 0 to 62 and 64 stations, mean values 14 and 22). Our small peculiar cluster is not detected by the dynsbm method.

To conclude this section we mention that the same dataset is analysed in Guigourès et al. (2015) with a different perspective. Randriamanamihaga et al. (2014) use a completely different approach, relying on Poisson mixture models on a similar dataset of origin/destination flows (bike sharing system in Paris). Their approach does not take into account the network structure of the data (where e.g. two flows from the same station are related). As a consequence, clusters are obtained on pairs of stations from which interpretation is completely different and in a way less natural.

\subsection{Enron dataset}

The Enron corpus contains emails exchanges among people working at Enron, mostly in the senior management, covering the period of the affair that led to the bankruptcy of the company (Klimt \& Yang, 2004). From the data provided by the CALO Project (2015) we extracted 21, 267 emails exchanged among 147 persons between 27 April, 2000 and 14 June, 2002, for which the sender, the recipient and the time when the email was sent are known. For most persons their position in the company is known: one out of four are employees, all other positions involve responsibility and we 

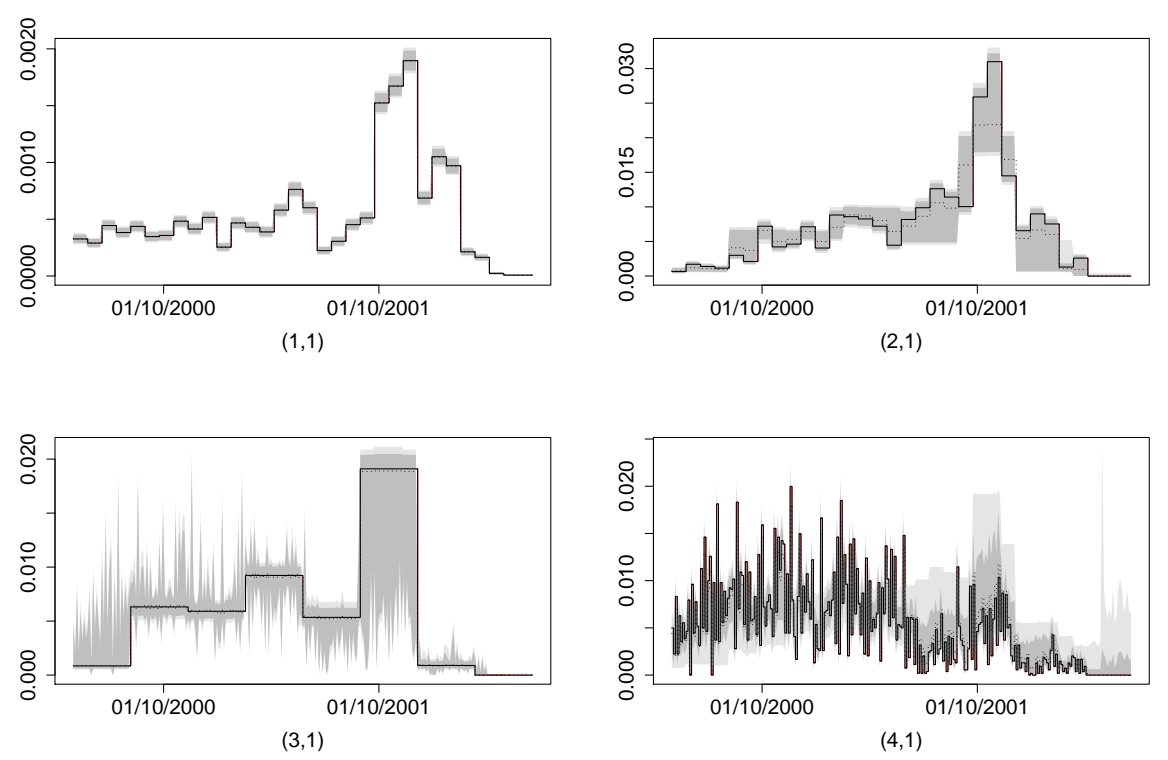

Figure 4: Enron: intensity estimates for pair of groups $(q, 1)$ for $q \in\{1, \ldots, 4\}$ and bootstrap confidence interval with confidence level $90 \%$ (lightgrey) and $80 \%$ (drakgrey).

summarize them as managers.

We applied our algorithm using the directed model and the histogram approach with regular dyadic partitions of maximal size $256\left(d_{\max }=8\right)$. For every fixed number of groups $Q \in$ $\{2, \ldots, 20\}$ the algorithm identifies one large group always containing the same individuals. One may consider this group to have the standard behaviour at the company. For varying values of $Q$ the differences in the clustering only concern the remaining individuals with non standard behaviour. The integrated classification likelihood criterion does not provide a reasonably small enough number of groups that could be used for interpretation. We thus choose to analyse the data with $Q=4$ clusters. The group with the standard behaviour (group 1) contains 127 people both employees and managers. The second largest group (group 4) contains almost exclusively managers, and group 2 is composed of employees. The standard behaviour (group 1) consists in little sending activity, but receiving mails from all other groups. The members of group 3 have the most intense email exchange. The specific manager and employee groups (groups 2 and 4 ) are quite similar and indeed at $Q=3$ both groups are merged together. For a number of pairs of groups communication is relatively constant over time, but for others the activity evolves a lot over time, as for instance for emails sent to members of group 1 (Figure 4). The intra-group intensity of group 1 is increasing with a peak in October 2001, which coincides with the beginning of the investigations related to the scandal. In contrast, the managers of group 4 communicate more intensely with group 1 during the first half of the observation time than during the last half. More details are provided in the Supplementary Material.

The bootstrap confidence intervals in Figure 4 are obtained via parametric bootstrap. In general, confidence intervals for large groups are very satisfactory, but this does not hold for small groups. Indeed, here some of the estimated probabilities $\hat{\pi}_{k}$ of group membership are very small (two are lower than $3 \%$ ), so bootstrap samples tend to have empty groups. Evidently, this has a devastating 
effect on the associated bootstrap intervals.

Rastelli et al. (2017) analyse the same dataset with a discrete-time model where individuals are allowed to change groups over time. They also obtain that most individuals are gathered in one group, which are rather inactive but receive some emails, and observe a specific behaviour of some groups of employees and managers. However, there is a difference in interpretation of results in the two models. In Rastelli's model groups may be identified with specific tasks like sending newsletters for example and individuals may execute this task during a while and then change activity. In contrast, our model identifies groups of individuals with a similar behaviour over the entire observation period. Our approach thus is natural for the analysis of the temporal evolution of the activity of a fixed group of persons.

Finally, we carried out a comparison with a classical stochastic block model. Indeed, taking $d_{\max }=0$ in our approach amounts to forget the timestamps of the emails, as the algorithm then only considers email counts over the whole observation period and our method boils down to a classical stochastic block model with Poisson emission distribution and mean parameter $A^{(q, l)}(T)$ (see for instance Mariadassou et al., 2010). Comparing the classification at $Q=4$ to the one obtained with our continuous-time approach, the adjusted rand index equals 0.798 indicating that the clusterings are different. Indeed, both models identify roughly the same large group with standard behaviour, but huge differences appear in the classification of the non standard individuals. This illustrates that our approach does effectively take into account the temporal distribution of the events to cluster the individuals. And the higher value of the complete log-likelihood in the Poisson process stochastic block model indicates that the solution is indeed an improvement to the classical model.

\section{Acknowledgment}

We would like to thank Agathe Guilloux for pointing out valuable references, Nathalie Eisenbaum for her help on doubly stochastic counting processes and Pierre Latouche for sharing information on datasets. The computations were partly performed at the institute for computing and data sciences at University Pierre and Marie Curie.

\section{Supplementary Material}

Supplementary material available online at Biometrika includes a complementary version of the model that specifically accounts for sparse datasets, the proofs of all theoretical results, technical details on the methods and algorithm, some supporting results for the analysis of the two datasets explored here, as well as the study of a third dataset. A zip file containing the computer code in $\mathrm{R}$ and the analysis of the datasets is available at http://cmatias.perso.math.cnrs.fr/ Docs/ppsbm_files.tgz

\section{References}

Allman, E., Matias, C. \& Rhodes, J. (2009). Identifiability of parameters in latent structure models with many observed variables. Ann. Statist. 37, 3099-3132.

Allman, E., Matias, C. \& Rhodes, J. (2011). Parameters identifiability in a class of random graph mixture models. J. Stat. Plan. Inference 141, 1719-1736. 
Andersen, P. K., Borgan, Ø., Gill, R. D. \& Keiding, N. (1993). Statistical models based on counting processes. Springer Series in Statistics. Springer-Verlag, New York.

BARAUD, Y. \& BIRGÉ, L. (2009). Estimating the intensity of a random measure by histogram type estimators. Probab. Theory Related Fields 143, 239-284.

Bickel, P. J., ChEn, A. \& LevinA, E. (2011). The method of moments and degree distributions for network models. Ann. Statist. 39, 2280-2301.

Biernacki, C., CeleuX, G. \& Govaert, G. (2000). Assessing a mixture model for clustering with the integrated completed likelihood. IEEE Trans. Pattern Anal. Machine Intel. 22, 719-725.

Blundell, C., Beck, J. \& Heller, K. A. (2012). Modelling reciprocating relationships with hawkes processes. In Advances in Neural Information Processing Systems 25, F. Pereira, C. J. C. Burges, L. Bottou \& K. Q. Weinberger, eds. Curran Associates, Inc., pp. 2600-2608.

BÖHNING, D. (1995). A review of reliable maximum likelihood algorithms for semiparametric mixture models. J. Stat. Plan. Inference 47, 5 - 28.

Bordes, L., Chauveau, D. \& Vandekerkhove, P. (2007). A stochastic EM algorithm for a semiparametric mixture model. Comput. Stat. Data Anal. 51, 5429 - 5443.

ButTs, C. T. (2008). A relational event framework for social action. Sociol. Methodol. 38, 155200.

CALO Project (2015). https://www.cs.cmu.edu/\$ \sim\$./enron/

Celisse, A., Daudin, J.-J. \& Pierre, L. (2012). Consistency of maximum-likelihood and variational estimators in the Stochastic Block Model. Electron. J. Statist. 6, 1847-1899.

Cho, Y.-S., Galstyan, A., Brantingham, P. J. \& Tita, G. (2014). Latent self-exciting point process model for spatial-temporal networks. Discrete Continuous Dyn. Syst. Ser. B 19, $1335-1354$.

Corneli, M., Latouche, P. \& Rossi, F. (2016). Exact ICL maximization in a non-stationary temporal extension of the stochastic block model for dynamic networks. Neurocomputing 192, $81-91$.

Dannemann, J. (2012). Semiparametric Hidden Markov models. J. Comput. Graph. Statist. 21, $677-692$.

Daudin, J.-J., PiCARD, F. \& Robin, S. (2008). A mixture model for random graphs. Statist. Comput. 18, 173-183.

Dempster, A. P., Laird, N. M. \& Rubin, D. B. (1977). Maximum likelihood from incomplete data via the EM algorithm. J. Roy. Statist. Soc. Ser. B 39, 1-38.

DuBois, C., ButTs, C. T. \& Smyth, P. (2013). Stochastic blockmodeling of relational event dynamics. In AISTATS, vol. 31. JMLR Workshop and Conference Proceedings. 
Fox, E. W., Short, M. B., Schoenberg, F. P., Coronges, K. D. \& Bertozzi, A. L. (2016). Modeling e-mail networks and inferring leadership using self-exciting point processes. J Am Stat Assoc 111, 564-584.

GrÉGOIRE, G. (1993). Least squares cross-validation for counting process intensities. Scand. J. Statist. 20, pp. 343-360.

Guigourès, R., Boullé, M. \& Rossi, F. (2015). Discovering patterns in time-varying graphs: a triclustering approach. Adv. Data Anal. Classif. , 1-28.

Holme, P. (2015). Modern temporal network theory: a colloquium. Eur. Phys. J. B 88, 234.

Hubert, L. \& Arabie, P. (1985). Comparing partitions. J. Classif. 2, 193-218.

Jordan, M., Ghahramani, Z., JaAkKola, T. \& Saul, L. (1999). An introduction to variational methods for graphical models. Mach. Learn. 37, 183-233.

Klimt, B. \& YAng, Y. (2004). The Enron Corpus: A new dataset for email classification research. In Machine Learning: ECML 2004, J.-F. Boulicaut, F. Esposito, F. Giannotti \& D. Pedreschi, eds., vol. 3201 of Lecture Notes in Computer Science. Springer Berlin Heidelberg.

Linderman, S. \& ADAMS, R. (2014). Discovering latent network structure in point process data. In Proceedings of the 31st International Conference on Machine Learning (ICML-14), E. P. Xing \& T. Jebara, eds., vol. 32.

Mariadassou, M., Robin, S. \& VACher, C. (2010). Uncovering latent structure in valued graphs: a variational approach. Ann. Appl. Stat. 4, 715-42.

Matias, C. \& Miele, V. (to appear). Statistical clustering of temporal networks through a dynamic stochastic block model. J. R. Stat. Soc. Ser. B. Stat. Methodol. Available at ArXiv:1506.07464.

Matias, C. \& Robin, S. (2014). Modeling heterogeneity in random graphs through latent space models: a selective review. Esaim Proc. \& Surveys 47, 55-74.

Perry, P. O. \& Wolfe, P. J. (2013). Point process modelling for directed interaction networks. J. R. Stat. Soc. Ser. B. Stat. Methodol. 75, 821-849.

RAMlaU-HANSEN, H. (1983). Smoothing counting process intensities by means of kernel functions. Ann. Statist. 11, pp. 453-466.

Randriamanamihaga, A. N., Côme, E., Oukhellou, L. \& Govaert, G. (2014). Clustering the Vélib' dynamic origin/destination flows using a family of Poisson mixture models. Neurocomputing 141, $124-138$.

Rastelli, R., Latouche, P. \& Friel, N. (2017). Choosing the number of groups in a latent stochastic block model for dynamic networks. Available at arXiv:1702.01418.

Reynaud-Bouret, P. (2006). Penalized projection estimators of the Aalen multiplicative intensity. Bernoulli 12, 633-661. 
Robin, S., BAr-Hen, A., Daudin, J.-J. \& Pierre, L. (2007). A semi-parametric approach for mixture models: Application to local false discovery rate estimation. Comput. Stat. Data Anal. $\mathbf{5 1 ,} 5483-5493$.

SNiJders, T. \& VAN DuiJn, M. (1997). Simulation for statistical inference in dynamic network models. In Simulating Social Phenomena, R. Conte, R. Hegselmann \& P. Terna, eds., vol. 456 of Lecture Notes in Economics and Mathematical Systems. Springer Berlin Heidelberg, pp. 493 512.

Snijders, T. A. B., Koskinen, J. \& Schweinberger, M. (2010). Maximum likelihood estimation for social network dynamics. Ann. Appl. Stat. 4, 567-588.

Stehlé, J., Voirin, N., Barrat, A., Cattuto, C., Isella, L., Pinton, J.-F. \& et Al. (2011). High-resolution measurements of face-to-face contact patterns in a primary school. PLoS ONE 6, e23176.

TRANSPORT FOR LONDON (2016). Cycle hire usage data 2012 - 2015. http://cycling.data.tfl.gov.uk/.

Vu, D. Q., Hunter, D., Smyth, P. \& Asuncion, A. U. (2011). Continuous-time regression models for longitudinal networks. In Adv Neural Inf Process Syst 24, J. Shawe-Taylor, R. Zemel, P. Bartlett, F. Pereira \& K. Weinberger, eds. Curran Associates, Inc., pp. 2492-2500.

WASSERman, S. (1980a). Analyzing social networks as stochastic processes. J Am Stat Assoc 75, 280-294.

WASSERMAN, S. (1980b). A stochastic model for directed graphs with transition rates determined by reciprocity. Sociol. Methodol. 11, pp. 392-412.

Xu, K. \& Hero, A. (2014). Dynamic stochastic blockmodels for time-evolving social networks. IEEE J. Sel. Topics Signal Process. 8, 552-562.

Yang, T., Chi, Y., Zhu, S., Gong, Y. \& Jin, R. (2011). Detecting communities and their evolutions in dynamic social networks—a Bayesian approach. Mach. Learn. 82, 157-189. 


\section{Supplementary material for: A semiparametric extension of the stochastic block model for longitudinal networks}

All the references are from the main manuscript, except for those appearing as S-xx that are within this document.

\section{S.1 Identifiability proofs}

Proof of Proposition 17 The proof follows ideas similar to those of Theorem 12 in Allman et al. (2011). For notational convenience this proof is presented in the undirected setup where the set of intensities is $\alpha=\left\{\alpha^{(q, l)}: q, l=1, \ldots, Q ; q \leq l\right\}$. The directed case is treated in the same way.

To explain the general idea of the proof we start by considering the distribution of one marginal process $N_{i, j}$. This is a Cox process directed by the random measure

$$
A_{i, j} \sim \sum_{q=1}^{Q} \sum_{l=1}^{Q} \pi_{q} \pi_{l} \delta_{A^{(q, l)}}
$$

Here for any $q \leq l$, we use the notation $A^{(q, l)}$ for the measure on $[0, T]$ defined by $A^{(q, l)}(I)=$ $\int_{I} \alpha^{(q, l)}(u) d u$ for all measurable $I \subset[0, T]$. We also recall that $\delta_{u}$ is the Dirac mass at point $u$. It is known that the mapping of probability laws of random measures into laws of Cox processes directed by them is a bijection (see for example Proposition 6.2.II in Daley and Vere-Jones, 2003). In other words the distribution of $N_{i, j}$ uniquely determines the finite measure (on the set of measures on $[0, T])$

$$
\sum_{q=1}^{Q} \sum_{l=1}^{Q} \pi_{q} \pi_{l} \delta_{A^{(q, l)}}
$$

According to Assumption 1 the intensities $\alpha^{(q, l)}$ are distinct. Hence, the corresponding measures $A^{(q, l)}$ are all different and we may recover from the distribution of our counting process $N_{i, j}$ the set of values $\left\{\left(\pi_{q}^{2}, A^{(q, q)}\right): q=1, \ldots, Q\right\} \cup\left\{\left(2 \pi_{q} \pi_{l}, A^{(q, l)}\right): q, l=1, \ldots, Q ; q<l\right\}$ or equivalently the set $\left\{\left(\pi_{q}^{2}, \alpha^{(q, q)}\right): q=1, \ldots, Q\right\} \cup\left\{\left(2 \pi_{q} \pi_{l}, \alpha^{(q, l)}\right) ; q, l=1, \ldots, Q ; q<l\right\}$. In particular we recover the functions $\alpha^{(q, l)}$ almost everywhere on $[0, T]$ up to a permutation on the pairs of groups $(q, l)$. However for the recovery up to a permutation in $\mathfrak{S}_{Q}$ it is necessary to consider higher-order marginals.

We now fix three distinct integers $i, j, k$ in $\{1, \ldots, n\}$ and consider the trivariate counting process $\left(N_{i, j}, N_{i, k}, N_{j, k}\right)$. In the same way, these are Cox processes directed by the triplet of random measures $\left(A_{i, j}, A_{i, k}, A_{j, k}\right)$ such that

$$
\left(A_{i, j}, A_{i, k}, A_{j, k}\right) \sim \sum_{q=1}^{Q} \sum_{l=1}^{Q} \sum_{m=1}^{Q} \pi_{q} \pi_{l} \pi_{m} \delta_{\left(A^{(q, l)}, A^{(q, m)}, A^{(l, m)}\right)} .
$$


We write this distribution in such a way that distinct components appear only once

$$
\begin{gathered}
\sum_{q=1}^{Q} \pi_{q}^{3} \delta_{\left(A^{(q, q)}, A^{(q, q)}, A^{(q, q)}\right)} \\
+\sum_{q=1}^{Q} \sum_{\substack{l=1 \\
l \neq q}}^{Q} \pi_{q}^{2} \pi_{l}\left\{\delta_{\left(A^{(q, q)}, A^{(q, l)}, A^{(q, l)}\right)}+\delta_{\left(A^{(q, l)}, A^{(q, q)}, A^{(q, l)}\right)}+\delta_{\left(A^{(q, l)}, A^{(q, l)}, A^{(q, q)}\right)}\right\} \\
+\sum_{q=1}^{Q} \sum_{\substack{l=1 \\
l \neq q}}^{Q} \sum_{\substack{m=1 \\
m \neq q}}^{Q} \pi_{q} \pi_{l} \pi_{m} \delta_{\left(A^{(q, l)}, A^{(q, m)}, A^{(l, m)}\right)} .
\end{gathered}
$$

Using the same reasoning we identify the triplets of values $\left\{\left(A^{(q, l)}, A^{(q, m)}, A^{(l, m)}\right): q, l, m=\right.$ $1, \ldots, Q\}$ up to a permutation on the triplets $(q, l, m)$. Among these, the only values with three identical components are $\left\{\left(A^{(q, q)} ; A^{(q, q)} ; A^{(q, q)}\right): q=1, \ldots, Q\right\}$ and thus the measures $\left\{A^{(q, q)}\right.$ : $q=1, \ldots, Q\}$ are identifiable up to a permutation in $\mathfrak{S}_{Q}$. Going back to $(\mathbf{S . 1})$ and looking for the Dirac terms at points that have two identical components (of the form $\left(A^{(q, q)}, A^{(q, l)}, A^{(q, l)}\right)$ and two other similar terms with permuted components), we can now identify the set of measures

$$
\left\{\left(A^{(q, q)},\left\{A^{(q, l)}: l=1, \ldots, Q ; l \neq q\right\}\right): q=1, \ldots, Q\right\} .
$$

This is equivalent to saying that we identify the measures $\left\{A^{(q, l)}: q, l=1, \ldots, Q ; q \leq l\right\}$ up to a permutation in $\mathfrak{S}_{Q}$. Obviously this also identifies the corresponding intensities $\left\{\alpha^{(q, l)}: q, l=\right.$ $1, \ldots, Q ; q \leq l\}$ almost everywhere on $[0, T]$ up to a permutation in $\mathfrak{S}_{Q}$. To finish the proof we need to identify the proportions $\pi_{q}$. Note that as we identified the components $\left\{A^{(q, q)}: q=\right.$ $1, \ldots, Q\}$, we recover from $\left[\right.$ S.1 the set of values $\left\{\pi_{q}^{3}: q=1, \ldots, Q\right\}$ up to the same permutation as on the $A^{(q, q)}$ 's. This concludes the proof.

Proof of Proposition 2. Note that the setup considered here is undirected. We follow some of the arguments already appearing in the proof of Proposition 1. Let $A^{\text {in }}$ (resp. $A^{\text {out }}$ ) denote the measure whose intensity is $\alpha^{\text {in }}$ (resp. $\alpha^{\text {out }}$ ). The univariate process $N_{i, j}$ is a Cox process directed by the random measure $A_{i, j}$ that is now distributed as

$$
A_{i, j} \sim\left(\sum_{q=1}^{Q} \pi_{q}^{2}\right) \delta_{A^{\text {in }}}+\left(\sum_{q=1}^{Q} \sum_{\substack{l=1 \\ l \neq q}}^{Q} \pi_{q} \pi_{l}\right) \delta_{A^{\text {out }}} .
$$

Thus the measures $A^{\text {in }}$ and $A^{\text {out }}$ are identifiable from the distribution of $N_{i, j}$, but only up to a permutation. Similarly to the previous proof we rather consider the trivariate Cox processes $\left(N_{i, j}, N_{i, k}, N_{j, k}\right)$ directed by the random measures $\left(A_{i, j}, A_{i, k}, A_{j, k}\right)$ whose distribution in the affiliation case has now five atoms

$$
\begin{aligned}
\left(\sum_{q=1}^{Q} \pi_{q}^{3}\right) \delta_{\left(A^{\text {in }}, A^{\text {in }}, A^{\text {in }}\right)} & +\left(\sum_{q=1}^{Q} \sum_{\substack{l=1 \\
l \neq q}}^{Q} \pi_{q}^{2} \pi_{l}\right) \delta_{\left(A^{\text {in }}, A^{\text {out }}, A^{\text {out }}\right)}+\left(\sum_{q=1}^{Q} \sum_{\substack{l=1 \\
l \neq q}}^{Q} \pi_{q}^{2} \pi_{l}\right) \delta_{\left(A^{\text {out }}, A^{\text {in }}, A^{\text {out }}\right)} \\
+ & \left(\sum_{q=1}^{Q} \sum_{\substack{l=1 \\
l \neq q}}^{Q} \pi_{q}^{2} \pi_{l}\right) \delta_{\left(A^{\text {out }}, A^{\text {out }}, A^{\text {in }}\right)}+\left(\sum_{q=1}^{Q} \sum_{\substack{l=1 \\
l \neq q}}^{Q} \sum_{\substack{m=1 \\
m \neq q, l}}^{Q} \pi_{q} \pi_{l} \pi_{m}\right) \delta_{\left(A^{\text {out }}, A^{\text {out }}, A^{\text {out }}\right)} .
\end{aligned}
$$


As previously, these five components are identifiable up to a permutation on $\mathfrak{S}_{5}$. Now it is easy to identify the three components for which two marginals have same parameters and the third one has a different parameter. Thus we recover exactly the measures $A^{\text {in }}$ and $A^{\text {out }}$. This also identifies the corresponding intensities $\alpha^{\text {in }}$ and $\alpha^{\text {out }}$ almost everywhere on $[0, T]$.

Now identification of the proportions $\left\{\pi_{q}: q=1, \ldots, Q\right\}$ follows an argument already used in the proof of Theorem 13 in Allman et al. (2011) that we recall here for completeness. From the trivariate distribution of $\left(N_{i, j}, N_{i, k}, N_{j, k}\right)$ and the already recovered values $A^{\text {in }}$ and $A^{\text {out }}$, we identify the proportion $\sum_{q=1}^{Q} \pi_{q}^{3}$. Similarly for any $n \geq 1$, by considering the multivariate distribution of $\left(N_{i, j}\right)_{(i, j) \in \mathcal{R}}$, we can identify the Dirac mass at point $\left(A^{\text {in }}, \ldots, A^{\text {in }}\right)$ and thus its weight which is equal to $\sum_{q=1}^{Q} \pi_{q}^{n}$. By the Newton identities the values $\left\{\sum_{q=1}^{Q} \pi_{q}^{n}: n=1, \ldots, Q\right\}$ determine the values of elementary symmetric polynomials $\left\{\sigma_{n}\left(\pi_{1}, \ldots, \pi_{Q}\right): n=1, \ldots, Q\right\}$. These, in turn, are (up to sign) the coefficients of the monic polynomial whose roots (with multiplicities) are precisely $\left\{\pi_{q}: q=1, \ldots, Q\right\}$. Thus the proportion parameters are recovered up to a permutation.

\section{S.2 Variational E-step: Proof of Proposition 3}

For the Kullback-Leibler divergence we compute

$$
\begin{gathered}
\mathrm{KL}\left\{\operatorname{pr}_{\tau}(\cdot \mid \mathcal{O}) \| \operatorname{pr}_{\theta}(\cdot \mid \mathcal{O})\right\}=E_{\tau}\left\{\log \frac{\operatorname{pr}_{\tau}(\mathcal{Z} \mid \mathcal{O})}{\operatorname{pr}_{\theta}(\mathcal{Z} \mid \mathcal{O})} \mid \mathcal{O}\right\}=E_{\tau}\left\{\log \frac{\operatorname{pr}_{\tau}(\mathcal{Z} \mid \mathcal{O}) \operatorname{pr}_{\theta}(\mathcal{O})}{\mathcal{L}(\mathcal{O}, \mathcal{Z} \mid \theta)} \mid \mathcal{O}\right\} \\
=\sum_{i=1}^{n} E_{\tau}\left(\log \tau^{i, Z_{i}} \mid \mathcal{O}\right)+\log \operatorname{pr}_{\theta}(\mathcal{O})-E_{\tau}\{\log \mathcal{L}(\mathcal{O}, \mathcal{Z} \mid \theta) \mid \mathcal{O}\}
\end{gathered}
$$

The complete-data $\log$-likelihood $\log \mathcal{L}(\mathcal{O}, \mathcal{Z} \mid \theta)$ is

$$
-\sum_{q=1}^{Q} \sum_{l=1}^{Q} Y_{\mathcal{Z}}^{(q, l)} A^{(q, l)}(T)+\sum_{q=1}^{Q} \sum_{l=1}^{Q} \sum_{m=1}^{M} Z_{m}^{(q, l)} \log \left\{\alpha^{(q, l)}\left(t_{m}\right)\right\}+\sum_{i=1}^{n} \sum_{q=1}^{Q} Z^{i, q} \log \pi_{q},
$$

where $Y_{\mathcal{Z}}^{(q, l)}$ and $Z_{m}^{(q, l)}$ have been introduced in Equations (1) and (3), respectively. Now note that $E_{\tau}\left(Z^{i, q} \mid \mathcal{O}\right)=\operatorname{pr}_{\tau}\left(Z^{i, q}=1 \mid \mathcal{O}\right)=\operatorname{pr}_{\tau}\left(Z_{i}=q \mid \mathcal{O}\right)=\tau^{i, q}$. Moreover by the factorised form of $\mathrm{pr}_{\tau}$, for every $i \neq j$ we have

$$
E_{\tau}\left(Z^{i, q} Z^{j, l} \mid \mathcal{O}\right)=E_{\tau}\left(Z^{i, q} \mid \mathcal{O}\right) E_{\tau}\left(Z^{j, l} \mid \mathcal{O}\right)=\tau^{i, q} \tau^{j, l}
$$

The quantity $Y^{(q, l)}$ is thus equal to $E_{\tau}\left(Y_{\mathcal{Z}}^{(q, l)} \mid \mathcal{O}\right)$, namely the variational approximation of the mean number of dyads with latent groups $(q, l)$. Similarly $\tau_{m}^{(q, l)}$ equals $E_{\tau}\left(Z_{m}^{(q, l)} \mid \mathcal{O}\right)$, the variational approximation of the probability that observation $\left(t_{m}, i_{m}, j_{m}\right)$ corresponds to a dyad with latent groups $(q, l)$. It follows that

$$
\hat{\tau}=\underset{\tau \in \mathcal{T}}{\operatorname{Argmin}} \operatorname{KL}\left\{\operatorname{pr}_{\tau}(\cdot \mid \mathcal{O}) \| \operatorname{pr}_{\theta}(\cdot \mid \mathcal{O})\right\}=\underset{\tau \in \mathcal{T}}{\operatorname{Argmax}} J(\theta, \tau),
$$

where $J(\theta, \tau)$ is

$$
-\sum_{q=1}^{Q} \sum_{l=1}^{Q} Y^{(q, l)} A^{(q, l)}(T)+\sum_{q=1}^{Q} \sum_{l=1}^{Q} \sum_{m=1}^{M} \tau_{m}^{(q, l)} \log \left\{\alpha^{(q, l)}\left(t_{m}\right)\right\}+\sum_{i=1}^{n} \sum_{q=1}^{Q} \tau^{i, q} \log \left(\frac{\pi_{q}}{\tau^{i, q}}\right) .
$$


The variational E-step consists in maximizing $J$ with respect to the $\tau^{i, q}$ 's which are constrained to satisfy $\sum_{q=1}^{Q} \tau^{i, q}=1$ for all $i$. In other words we maximize

$$
M(\tau, \gamma)=J(\theta, \tau)+\sum_{i=1}^{n} \gamma_{i}\left(\sum_{q=1}^{Q} \tau^{i, q}-1\right),
$$

with Lagrange multipliers $\gamma_{i}$. The partial derivatives are

$$
\begin{aligned}
\frac{\partial}{\partial \tau^{i, q}} M(\tau, \gamma)= & -\sum_{l=1}^{Q} \sum_{j \neq i} \tau^{j, l}\left\{A^{(q, l)}(T)+A^{(l, q)}(T)\right\}+\sum_{l=1}^{Q} \sum_{m=1}^{M} \mathbb{1}_{\left\{i_{m}=i\right\}} \tau^{j_{m}, l} \log \left\{\alpha^{(q, l)}\left(t_{m}\right)\right\} \\
& +\sum_{l=1}^{Q} \sum_{m=1}^{M} \mathbb{1}_{\left\{j_{m}=i\right\}} \tau^{i_{m}, l} \log \left\{\alpha^{(l, q)}\left(t_{m}\right)\right\}+\log \left(\frac{\pi_{q}}{\tau^{i, q}}\right)-1+\gamma_{i}, \\
\frac{\partial}{\partial \gamma_{i}} M(\tau, \gamma)= & \sum_{q=1}^{Q} \tau^{i, q}-1 .
\end{aligned}
$$

The partial derivatives are null if and only if $\sum_{q=1}^{Q} \tau^{i, q}=1$ and the $\tau^{i, q}$ 's satisfy the fixed point equations (6), with $\exp \left(\gamma_{i}-1\right)$ being the normalizing constant.

\section{S.3 Details on the Algorithm}

Initialisation is a crucial point for any clustering method. Our variational expectation-maximization algorithm starts with a classification of the nodes and iterates an M-step followed by a variational E-step. We apply the algorithm on multiple initial classifications of the nodes based on various aggregations of the data: on the whole time interval and on sub-intervals. Sub-intervals are obtained through regular dyadic partitions of $[0, T]$ (parameter 1 . part in the R code) and a $k$-means algorithm applied on the rows of the adjacency matrices of each of the aggregated datasets provides starting points for our algorithm. To obtain further starting values we use perturbations of the different $k$-means classifications: a given percentage of the total number of individuals is picked at random (parameter perc.perturb) and their group memberships are shuffled. The perturbation procedure can be applied several times (parameter $n$. perturb). The algorithm returns as final result the run that achieves the largest value of criterion $J$.

There is no theoretical result on the existence of a solution to the fixed point equation (6). The iterations for the fixed point equation are initialized with the value of $\tau$ obtained at the previous variational E-step. In practice, convergence is fast and we stop the fixed-point iterations either when convergence is achieved $\left(\left|\tau^{[s]}-\tau^{[s-1]}\right|<\varepsilon=10^{-6}\right)$ or when the maximal number of iterations is attained (fix. iter $=10$ ).

As the variational expectation-maximization algorithm aims at maximizing $J$ defined in (S.2), the algorithm is stopped when the increase of $J$ is less than a given threshold $\left(\varepsilon=10^{-6}\right)$, that is when

$$
\left|\frac{J\left(\theta^{[s+1]}, \tau^{[s+1]}\right)-J\left(\theta^{[s]}, \tau^{[s]}\right)}{J\left(\theta^{[s]}, \tau^{[s]}\right)}\right|<\varepsilon,
$$

or when the maximal number of iterations has been attained (nb. iter=50). 


\section{S.4 Additional tables and figures}

Figure S.1 shows the intensities used in Scenario 1 from the synthetic experiments to assess the clustering performances of our method.

Table 2 gives the risks $\operatorname{RISK}(q, l)$ and standard deviations of the histogram and the kernel versions of our method as well as oracle quantities (obtained with known group labels) in Scenario 2 when $n=20$ (this is the analogue of Table 1 in the main manuscript where $n=50$ ).

Figure $\mathrm{S} .2$ shows boxplots of the adjusted rand index obtained from the synthetic experiments from Scenario 2. They are computed over 1000 datasets with different numbers $n$ of individuals and for the two estimation methods (histogram and kernel).

Figure $\mathrm{S.3}$ shows the model selection results on the number of groups based on the integrated classification likelihood in Scenario 2 with $n=20$ : the left panel shows the frequency of the selected values $\hat{Q}$ over the 1000 datasets; the right panel shows boxplots of the adjusted rand index between the estimated classification with 3 groups and the true latent structure as a function of the number of groups selected by the integrated classification likelihood criterion. On this right panel, one can see that when the criterion does not select the correct number $Q$ the adjusted rand index of the classification with three groups is rather low indicating that the algorithm has failed in the classification task and probably only a local maximum of the criterion $J$ has been found.

Turning to the London bike sharing system dataset, Figure S.4 shows the temporal profiles of the 2 stations in the smallest cluster for day 1 . One can see that these are 'outgoing' stations around $8 \mathrm{am}$ and 'incoming' stations between 5 and $7 \mathrm{pm}$. Figure S.5 shows the highest intensities estimated by our model between these 6 clusters, all other intensities are almost null. The most important interactions occur from cluster 4 (the smallest cluster) to cluster 5 ('City of London' cluster) in the morning and conversely from cluster 5 to cluster 4 at the end of the day.

Concerning the analysis of the Enron dataset we provide here additional tables and figures for $Q=4$ groups. Table 3 gives the size and composition of the four groups. For a part of the persons in the dataset the position at Enron is not available. This is the reason why the total size of the group sometimes exceeds the sum of the number of managers and employees in the group.

Figure S.6 gives the logarithm of the mean values of the estimated intensities $\alpha^{(q, l)}$. The lack of symmetry of the matrix indicates that communication is far from being symmetric and that the use of the directed model is appropriate.

Finally, Figure S.7 shows the estimated intensities and associated bootstrap confidence intervals. The bootstrap intervals are obtained by parametric bootstrap. More precisely, every bootstrap sample contains the same number of individuals (here $n=147$ ) and for every individual $i$ the group membership $Z_{i}^{*}$ is drawn from the multinomial distribution $\mathcal{M}(1, \hat{\pi})$ where $\hat{\pi}$ is the vector of group probabilities estimated from the data. Then for every pair of individuals $(i, j)$ realizations from a Poisson process with intensity $\hat{\alpha}^{\left(Z_{i}^{*}, Z_{j}^{*}\right)}$ are simulated, where $\left\{\hat{\alpha}^{(q, l)}\right\}_{q, l=1, \ldots, Q}$ denote the estimated intensities. Finally, bootstrap confidence intervals are obtained by the percentile method.

Here the bootstrap intervals suffer from the fact that some of the group probabilities $\hat{\pi}_{k}$ are very low, implying that the probability that a bootstrap sample contains empty groups is relatively high (0.15). That is, about $15 \%$ of the bootstrap samples do not provide any information on some of the intensities $\alpha^{(q, l)}$, implying that the associated estimators are completely erroneous. The groups that are the most concerned by the problem are group 2 and 3 . 


\section{S.5 Additional example: Primary school temporal network dataset}

To understand contacts between children at school and to quantify the transmission opportunities of respiratory infections, data on face-to-face interactions in a French primary school were collected. The dataset is presented in detail in Stehlé et al. (2011) and available online (SocioPatterns, 2015). Children are aged from 6 to 12 years and the school is composed of five grades, each of them comprising two classes, for a total of 10 classes (denoted by $1 A, 1 B, \ldots, 5 A, 5 B$ ). Each class has an assigned teacher and an assigned room. The school day runs from $8.30 \mathrm{am}$ to $4.30 \mathrm{pm}$, with a lunch break from $12 \mathrm{pm}$ to $2 \mathrm{pm}$ and two breaks of $20-25 \mathrm{~min}$ in the morning and in the afternoon. Lunch is served in a common canteen and a shared playground is located outside the main building. As the playground and the canteen do not have enough capacity to host all pupils at a time, only two or three classes have breaks together, and lunch is served in two turns. The dataset contains 125,773 face to face contacts among $n=242$ individuals ( 232 children and 10 teachers) observed during two days. We applied our procedure in the undirected setup with histograms based on a regular dyadic partitions with maximum size $256\left(d_{\max }=8\right)$.

The integrated classification likelihood criterion achieves its maximum with $\hat{Q}=17$ latent groups. Figure $\mathrm{S.8}$ shows the clustering of the $n$ individuals into the 17 groups, where children from different classes are represented with different colors. Some groups correspond exactly or almost exactly to classes (for example, group 9 consists almost perfectly of class 1A), whereas other classes are split into several groups (for example class 1B is splited into groups 1 and 16). Moreover, one group (group 6) corresponds to the entire class 4B with, in addition, pupils coming from almost all other classes. Teachers never form a particular group apart, but they are generally in the cluster of their assigned class.

The highest intensities are the intra-group intensities. As groups mainly correspond to classes, this highlights that most contacts involve individuals of the same class and that the dataset is structured into communities (i.e. groups of highly connected individuals and with few inter-groups interactions). Figure S.9 shows the estimated intra-group intensities for each group with at least 3 individuals. Peaks of interactions are observed during the two breaks in the morning and in the afternoon. At lunch time interactions between children vary from the first to the second day and are less important than during the breaks when they play together. We also observe periods with no interaction at all. For example, the estimated intensity of group 9 (class 1A) is null between 3:30am and 4am suggesting that some particular school activity like sports takes place during which contacts were not observable for technical reasons. The group number 6 composed with the entire class 4B and others pupils clearly appears as the group with the lowest intra-group intensity. This means that this cluster gathers the individuals having less interactions with others. Class 4B also appears as the class having the least intra-class interactions in Stehlé et al.(2011).

Concerning inter-group connections most of the estimated intensities for groups $(q, l)$ with $q \neq l$ can be considered as null, except for some that we discuss now. First, as our procedure splits some children of the same class into separate groups, the inter-group interactions associated with these clusters correspond in fact to intra-class interactions. For example, class 1B is split into group 1 (with 18 pupils) and group 16 (with 7 pupils). The estimated inter-group intensity shows that those two groups interact. Our clustering has formed two separate groups because group 1 has more intra-group interactions than the other (see Figure S.9.

Second, intensities between groups made of children of the same grade are significant, suggesting that children mostly interact with children of the same age (see e.g. Figure S.10 that shows the case $(q, l)=(5,13))$. Those interactions are observed during the two breaks in the morning and in 
the afternoon as well as at lunch time.

Third, the estimated intensities suggest particular behaviour of some pupils. For example, concerning class $2 \mathrm{~B}$ (except the two pupils assigned to group 6), which is separated into group 12 (with 21 pupils), group 11 (with 2 pupils) and group 17 (with only one pupil), the estimated intensities (see Figure S.11) suggest first that the two children in group 11 have very strong interaction with the pupil in group 17 (notice the different $y$-scale used in the Figure), and second that those interactions do not occur during the lunch time.

Similar results are obtained for classes $2 \mathrm{~A}$ and $5 \mathrm{~B}$. This means that our procedure detects subgroups of pupils with a specific behaviour.

\section{S.6 The sparse setup: theory}

We consider an extended setup where some of the processes $N_{i, j}$ may have a null intensity. We thus introduce additional latent variables $U_{i, j} \in\{0,1\},((i, j) \in \mathcal{R})$ that conditional on the $Z_{i}$ 's are independent Bernoulli with $\beta_{q, l}$ being the parameter of the distribution of $U_{i, j}$ conditional on $Z^{i, q} Z^{j, l}=1$. We keep the global conditional independence assumption by imposing that conditional on $\left(Z_{i}, U_{i, j}\right)_{(i, j) \in \mathcal{R}}$ the counting processes $\left(N_{i, j}\right)_{(i, j) \in \mathcal{R}}$ are independent. Then conditional on $U_{i, j}, Z_{i}, Z_{j}$, the counting process $N_{i, j}$ is an inhomogeneous Poisson process with intensity

$$
U_{i, j} \alpha^{Z_{i}, Z_{j}}=\sum_{q, l=1}^{Q} U_{i j} Z^{i, q} Z^{j, l} \alpha^{(q, l)}, \quad((i, j) \in \mathcal{R}) .
$$

In this way the additional latent variable $U_{i, j}$ accounts for sparsity in the interaction processes; in each pair of groups $(q, l)$, there is now a proportion $1-\beta_{q, l}$ of dyads $(i, j) \in \mathcal{R}$ that do not interact (so that the corresponding process $N_{i, j}$ is almost surely 0 ). As such these non interacting dyads will not tend to decrease the estimate of the common intensity $\alpha^{(q, l)}$. Moreover clustering in this model should give different groups, less driven by the absence of interactions.

We let $\mathcal{U}=\left(U_{i, j}\right)_{(i, j) \in \mathcal{R}}$ and the parameter value is $\theta=(\pi, \beta, \alpha)$.

Identifiability may be proved under the same assumptions, requiring moreover that none of the intensities $\alpha^{(q, l)}$ is itself equal to zero. We discuss this in the undirected case (similarly to the identifiability proof of the main model). Indeed $N_{i, j}$ is now a counting process directed by the random measure $A_{i, j}$ whose distribution is

$$
A_{i, j} \sim \sum_{q=1}^{Q} \sum_{l=1}^{Q} \pi_{q} \pi_{l}\left\{\beta_{q, l} \delta_{A^{(q, l)}}+\left(1-\beta_{q, l}\right) \delta_{0}\right\} .
$$

Fixing three distinct integers $i, j, k$ in $\{1, \ldots, n\}$ and considering the trivariate counting process $\left(N_{i, j}, N_{i, k}, N_{j, k}\right)$ we end up with the distribution of the triplet of random measures $\left(A_{i, j}, A_{i, k}, A_{j, k}\right)$. There the expressions become more cumbersome but the very same reasoning may be applied to identify the measures $\left\{A^{(q, l)}: q, l=1, \ldots, Q ; q \leq l\right\}$ up to a permutation in $\mathfrak{S}_{Q}$. Concerning identification of $\pi$ and $\beta$, we obtain the set of weights $\left\{\pi_{q}^{3} \beta_{q, q}^{3}: q=1, \ldots, Q\right\}$ that is attached to the $Q$ components corresponding to Dirac masses at points of the form $\left(A^{(q, q)}, A^{(q, q)}, A^{(q, q)}\right)$. Moreover we also obtain the set of weights $\left\{\pi_{q}^{3} \beta_{q, q}^{2}\left(1-\beta_{q, q}\right): q=1, \ldots, Q\right\}$ attached to Dirac masses at points $\left(A^{(q, q)}, A^{(q, q)}, 0\right)$. As the $A^{(q, q)}$ 's are unique we can match the value $\pi_{q}^{3} \beta_{q, q}^{3}$ with 
$\pi_{q}^{3} \beta_{q, q}^{2}\left(1-\beta_{q, q}\right)$ and thus obtain (through a simple ratio) $\beta_{q, q}$ and also $\pi_{q}$. In other words the sets $\left\{\beta_{q, q}: q=1, \ldots, Q\right\}_{q}$ and $\left\{\pi_{q}: q=1, \ldots, Q\right\}_{q}$ are identifiable. Finally we may look at the weights $\pi_{q}^{2} \pi_{l} \beta_{q, q} \beta_{q, l}^{2}$ associated with Dirac masses at points of the form $\left(A^{(q, q)}, A^{(q, l)}, A^{(q, l)}\right)$ with $q \neq l$. As the cumulative intensities $\left\{A^{(q, l)}: q, l=1, \ldots, Q ; q \leq l\right\}$ have been identified up to a permutation in $\mathfrak{S}_{Q}$, together with the pair of $\left(\pi_{q}, \beta_{q, q}\right)$ 's (for the same permutation), we obtain the values $\left\{\beta_{q, l}: q \neq l\right\}$.

Let us turn to inference of this model. To fix the notation we use the directed setup but similar equations may be derived in the undirected case. The complete-data likelihood is

$$
\begin{aligned}
\mathcal{L}_{\text {sparse }}(\mathcal{O}, \mathcal{Z}, \mathcal{U} \mid \theta)= & \mathcal{L}(\mathcal{O} \mid \mathcal{Z}, \mathcal{U}, \theta) \times \mathcal{L}(\mathcal{U} \mid \mathcal{Z}, \theta) \times \mathcal{L}(\mathcal{Z} \mid \theta) \\
= & \exp \left\{-\sum_{(i, j) \in \mathcal{R}} U_{i, j} A^{\left(Z_{i}, Z_{j}\right)}(T)\right\} \times\left[\prod_{m=1}^{M} \alpha^{\left(Z_{i_{m}}, Z_{j_{m}}\right)}\left(t_{m}\right)\right] \\
& \times\left[\prod_{(i, j) \in \mathcal{R}} \prod_{q=1}^{Q} \prod_{l=1}^{Q}\left\{\beta_{q, l}^{U_{i, j}}\left(1-\beta_{q, l}\right)^{1-U_{i, j}}\right\}^{Z^{i, q} Z^{j, l}}\right] \times\left[\prod_{i=1}^{n} \prod_{q=1}^{Q} \pi_{q}^{Z^{i, q}}\right] .
\end{aligned}
$$

The true conditional distribution of the latent variables $(\mathcal{Z}, \mathcal{U})$ given the observations writes

$$
\operatorname{pr}_{\theta}(\mathcal{Z}, \mathcal{U} \mid \mathcal{O})=\operatorname{pr}_{\theta}(\mathcal{Z} \mid \mathcal{O}) \operatorname{pr}_{\theta}(\mathcal{U} \mid \mathcal{Z}, \mathcal{O})=\operatorname{pr}_{\theta}(\mathcal{Z} \mid \mathcal{O}) \prod_{(i, j) \in \mathcal{R}} \operatorname{pr}_{\theta}\left(U_{i, j} \mid Z_{i}, Z_{j}, N_{i, j}\right)
$$

A main difference with the previous setting is that now this conditional distribution has two parts: the one concerning $\mathcal{U}$ has a factorised form and can thus be computed exactly, while the part concerning $\mathcal{Z}$ still has an intricate dependence structure and we rely on a variational approximation to deal with it. We thus introduce a new conditional factorised distribution $\tilde{\mathrm{pr}}_{\tau, \theta}(\cdot \mid \varnothing)$ on the variables $\mathcal{Z}, \mathcal{U}$ that depends on the observations $\varnothing$ and is defined as

$$
\begin{aligned}
& \tilde{\operatorname{pr}}_{\tau, \theta}\left(\mathcal{Z}=\left(q_{1}, \ldots, q_{n}\right), \mathcal{U}=\left(u_{i, j}\right)_{(i, j) \in \mathcal{R}} \mid \mathcal{O}\right) \\
& =\prod_{i=1}^{n} \operatorname{pr}_{\tau}\left(Z_{i}=q_{i} \mid \mathcal{O}\right) \prod_{(i, j) \in \mathcal{R}} \operatorname{pr}_{\theta}\left(U_{i, j}=u_{i, j} \mid Z_{i}=q_{i}, Z_{j}=q_{j}, N_{i, j}\right) \\
& =\prod_{i=1}^{n} \tau^{i, q_{i}} \prod_{(i, j) \in \mathcal{R}} \operatorname{pr}_{\theta}\left(U_{i, j}=u_{i, j} \mid Z_{i}=q_{i}, Z_{j}=q_{j}, N_{i, j}\right),
\end{aligned}
$$

for any $\left(q_{1}, \ldots, q_{n}\right) \in\{1, \ldots, Q\}^{n}$ and $\left(u_{i, j}\right)_{(i, j) \in \mathcal{R}} \in\{0,1\}^{r}$. As it does not depend on $\theta$, we let $\tilde{\mathrm{pr}} \tau(\mathcal{Z} \mid \varnothing)$ denote the marginal distribution on $\mathcal{Z}$ of the distribution $\tilde{\mathrm{pr}}_{\tau, \theta}(\cdot \mid \varnothing)$ and $\tilde{E}_{\tau}(\cdot \mid \varnothing)$ the corresponding expectation. Moreover, the true conditional distribution of $U_{i, j}$ is given by

$\operatorname{pr}_{\theta}\left(U_{i, j}=1 \mid Z_{i}=q, Z_{j}=l, N_{i, j}\right)=1\left\{N_{i, j}(T)>0\right\}+\rho_{\theta}(q, l) 1\left\{N_{i, j}(T)=0\right\}:=\rho_{\theta}(i, j, q, l)$, where $\rho_{\theta}(q, l)=\frac{\beta_{q, l} \exp \left\{-A^{(q, l)}(T)\right\}}{1-\beta_{q, l}+\beta_{q, l} \exp \left\{-A^{(q, l)}(T)\right\}}$.

Indeed, whenever we observe an interaction event between $(i, j)$ (namely $N_{i, j}(T)>0$ ) we know that $U_{i j}=1$ almost surely. Otherwise $\left(N_{i, j}(T)=0\right)$, we either have a null intensity process or a 
non-null intensity process with zero observations. Note that the parameters $\rho_{\theta}(q, l)$ (or equivalently the $\left.\rho_{\theta}(i, j, q, l)\right)$ are not additional variational parameters; these are just functions of the original parameter $\theta$. Finally we have

$$
\tilde{\mathbf{p r}}_{\tau, \theta}(\mathcal{Z}, \mathcal{U} \mid \mathcal{O})=\left\{\prod_{i=1}^{n} \tilde{\mathbf{p r}}_{\tau}\left(Z_{i} \mid \varnothing\right)\right\} \times \prod_{(i, j) \in \mathcal{R}} \rho_{\theta}\left(i, j, Z_{i}, Z_{j}\right)^{U_{i, j}}\left(1-\rho_{\theta}\left(i, j, Z_{i}, Z_{j}\right)\right)^{1-U_{i, j}}
$$

Let us now derive our variational approximation. Denoting by $\tilde{E}_{\tau, \theta}(\cdot \mid \varnothing)$ the expectation under the distribution $\tilde{\mathrm{pr}}_{\tau, \theta}(\cdot \mid \varnothing)$ on $(\mathcal{Z}, \mathcal{U})$ and by $\theta^{[s]}$ the current parameter value, we write as usual

$$
\begin{aligned}
& \log \operatorname{pr}_{\theta[s]}(\varnothing) \\
& =\tilde{E}_{\tau, \theta}\left\{\log \operatorname{pr}_{\theta[s]}(\varnothing, \mathcal{Z}, \mathcal{U}) \mid \varnothing\right\}-\tilde{E}_{\tau, \theta}\left\{\log \operatorname{pr}_{\theta[s]}(\mathcal{Z}, \mathcal{U} \mid \varnothing) \mid \varnothing\right\} \\
& =\tilde{E}_{\tau, \theta}\left\{\log \operatorname{pr}_{\theta[s]}(\varnothing, \mathcal{Z}, \mathcal{U}) \mid \varnothing\right\}+\mathcal{H}\left\{\tilde{p r}_{\tau, \theta}(\mathcal{Z}, \mathcal{U} \mid \varnothing)\right\}+\operatorname{KL}\left\{\tilde{p r}_{\tau, \theta}(\mathcal{Z}, \mathcal{U} \mid \varnothing) \| \operatorname{pr}_{\theta[s]}(\mathcal{Z}, \mathcal{U} \mid \varnothing)\right\}
\end{aligned}
$$

As a consequence, we introduce a new criterion $\tilde{J}\left(\tau, \theta ; \theta^{[s]}\right)$ that is a lower bound on the loglikelihood $\log \operatorname{pr}_{\theta[s]}(\varnothing)$ and defined as

$$
\begin{aligned}
\tilde{J}\left(\tau, \theta ; \theta^{[s]}\right)= & \tilde{E}_{\tau, \theta}\left\{\log \operatorname{pr}_{\theta[s]}(\varnothing, \mathcal{Z}, \mathcal{U}) \mid \varnothing\right\}+\mathcal{H}\left\{\tilde{p r}_{\tau, \theta}(\mathcal{Z}, \mathcal{U} \mid \mathcal{O})\right\} \\
= & -\sum_{(i, j) \in \mathcal{R}} \sum_{q=1}^{Q} \sum_{l=1}^{Q} \tau^{i, q} \tau^{j, l} \rho_{\theta}(i, j, q, l)\left\{A^{[s]}\right\}^{(q, l)}(T) \\
& +\sum_{q=1}^{Q} \sum_{l=1}^{Q} \sum_{m=1}^{M} \tau^{i{ }_{m}, q} \tau^{j_{m}, l} \log \left[\left\{\alpha^{[s]}\right\}^{(q, l)}\left(t_{m}\right)\right] \\
& +\sum_{(i, j) \in \mathcal{R}} \sum_{q=1}^{Q} \sum_{l=1}^{Q} \tau^{i, q} \tau^{j, l}\left[\rho_{\theta}(i, j, q, l) \log \beta_{q, l}^{[s]}+\left\{1-\rho_{\theta}(i, j, q, l)\right\} \log \left(1-\beta_{q, l}^{[s]}\right)\right] \\
& +\sum_{i=1}^{n} \sum_{q=1}^{Q} \tau^{i, q} \log \left\{\frac{\pi_{q}^{[s]}}{\tau^{i, q}}\right\}-\sum_{(i, j) \in \mathcal{R}} \sum_{q=1}^{Q} \sum_{l=1}^{Q} \tau^{i, q} \tau^{j, l} \psi\left(\rho_{\theta}(i, j, q, l)\right),
\end{aligned}
$$

where $\psi(\rho)=\rho \log \rho+(1-\rho) \log (1-\rho)$ is the entropy of the Bernoulli distribution with parameter $\rho$. Using the definition of $\rho_{\theta}(i, j, q, l)$ and $\psi(1)=0$, the last term in the right-hand side simplifies to

$$
\sum_{(i, j) \in \mathcal{R}} \sum_{q=1}^{Q} \sum_{l=1}^{Q} \tau^{i, q} \tau^{j, l} \psi\left(\rho_{\theta}(i, j, q, l)\right)=\sum_{q=1}^{Q} \sum_{l=1}^{Q} \psi\left(\rho_{\theta}(q, l)\right) \sum_{(i, j) \in \mathcal{R}} \tau^{i, q} \tau^{j, l} 1\left\{N_{i, j}(T)=0\right\} .
$$

The variational E-step consists in maximizing $\tilde{J}\left(\tau, \theta ; \theta^{[s]}\right)$ with respect to $(\tau, \theta)$. This is equivalent to choosing the variational distribution $\tilde{\mathrm{pr}}_{\tau, \theta}$ that minimises the Kullback-Leibler divergence $\mathrm{KL}\left\{\tilde{\operatorname{pr}}_{\tau, \theta}(\mathcal{Z}, \mathcal{U} \mid \varnothing) \| \operatorname{pr}_{\theta[s]}(\mathcal{Z}, \mathcal{U} \mid \varnothing)\right\}$. The solution in $\theta$ is naturally obtained for $\theta=\theta^{[s]}$.

We need to choose the variational parameter $\tau$ that maximizes $\tilde{J}\left(\tau, \theta^{[s]} ; \theta^{[s]}\right)$. Similarly to the non sparse setup we obtain that $\tau^{[s]}$ satisfies a fixed point equation in $\tau$,

$$
\tau^{i, q} \propto \pi_{q}^{[s]} \exp \left\{\tilde{D}_{i q}\left(\tau, \theta^{[s]}\right)\right\}, \quad(i=1, \ldots, n ; q=1, \ldots, Q),
$$


where

$$
\begin{aligned}
\tilde{D}_{i q}(\tau, \theta)= & -\sum_{l=1}^{Q} \sum_{\substack{j=1 \\
j \neq i}}^{n} \tau^{j, l}\left\{\rho_{\theta}(i, j, q, l) A^{(q, l)}(T)+\rho_{\theta}(j, i, l, q) A^{(l, q)}(T)\right\} \\
& -\sum_{l=1}^{Q} \psi\left(\rho_{\theta}(q, l)\right) \sum_{\substack{j=1 \\
j \neq i}}^{n} \tau^{j, l} 1\left\{N_{i, j}(T)=0\right\}-\sum_{l=1}^{Q} \psi\left(\rho_{\theta}(l, q)\right) \sum_{\substack{j=1 \\
j \neq i}}^{n} \tau^{j, l} 1\left\{N_{j, i}(T)=0\right\} \\
& +\sum_{l=1}^{Q} \sum_{m=1}^{M}\left[\mathbb{1}_{\left\{i_{m}=i\right\}} \tau^{j m, l} \log \left\{\alpha^{(q, l)}\left(t_{m}\right)\right\}+\mathbb{1}_{\left\{j_{m}=i\right\}} \tau^{i_{m}, l} \log \left\{\alpha^{(l, q)}\left(t_{m}\right)\right\}\right] \\
& +\sum_{l=1}^{Q} \sum_{\substack{j=1 \\
j \neq i}}^{n} \tau^{j, l}\left[\rho_{\theta}(i, j, q, l) \log \beta_{q, l}+\left\{1-\rho_{\theta}(i, j, q, l)\right\} \log \left(1-\beta_{q, l}\right)\right. \\
& \left.+\rho_{\theta}(j, i, l, q) \log \beta_{l, q}+\left\{1-\rho_{\theta}(j, i, l, q)\right\} \log \left(1-\beta_{l, q}\right)\right] .
\end{aligned}
$$

The M-step consists in maximizing $\tilde{J}\left(\tau^{[s]}, \theta^{[s]} ; \theta\right)$ with respect to $\theta$. It is again divided into two parts, treating the finite-dimensional parameter $(\pi, \beta)$ differently than the infinite dimensional one $\alpha$. We thus first maximize $\tilde{J}\left(\tau^{[s]}, \theta^{[s]} ; \pi, \beta, \alpha\right)$ with respect to $(\pi, \beta)$ using the current parameter value $\alpha=\alpha^{[s]}$. The solution with respect to $\pi$ is the same as in the non sparse case and given in (7). Now optimization with respect to $\beta$ leads to (denoting $\rho^{[s]}=\rho_{\theta[s]}$ ),

$$
\beta_{q, l}^{[s+1]}=\frac{\sum_{(i, j) \in \mathcal{R}}\left\{\tau^{[s]}\right\}^{i, q}\left\{\tau^{[s]}\right\}^{j, l} \rho^{[s]}(i, j, q, l)}{\sum_{(i, j) \in \mathcal{R}}\left\{\tau^{[s]}\right\}^{i, q}\left\{\tau^{[s]}\right\}^{j, l}}, \quad q, l=1, \ldots, Q .
$$

Then estimation of the intensities $\alpha^{(q, l)}$ is done exactly as previously, except that we replace the variational process $N^{(q, l)}$ by $\tilde{N}^{(q, l)}=\sum_{(i, j) \in \mathcal{R}} \rho^{[s]}(i, j, q, l)\left\{\tau^{[s]}\right\}^{i, q}\left\{\tau^{[s]}\right\}^{j, l} N_{i, j}$.

Finally we start from an initial value of the clusters $\mathcal{Z}$ (see Section $S .3$ ) that we treat as probabilities $\left\{\left(\tau^{i, q}\right)_{1 \leq q \leq Q} ; 1 \leq i \leq n\right\}$. Then we initialise the sparsity parameters $\beta_{q, l}$ and mean intensities $A^{(q, l)}(T)$ with

$$
\beta_{q, l}=\frac{\sum_{(i, j) \in \mathcal{R}} Z^{i, q} Z^{j, l} 1\left\{N_{i, j}(T)>0\right\}}{\sum_{(i, j) \in \mathcal{R}} Z^{i, q} Z^{j, l}}, \quad A^{(q, l)}(T)=\frac{\sum_{(i, j) \in \mathcal{R}} Z^{i, q} Z^{j, l} N_{i, j}(T)}{\sum_{(i, j) \in \mathcal{R}} Z^{i, q} Z^{j, l} 1\left\{N_{i, j}(T)>0\right\}} .
$$

This enables to initialise $\rho(i, j, q, l)$ with S.3. After these initialisations, we are ready to iterate the following steps. At iteration $s \geq 1$ we do

- M-step: Update $\pi^{[s+1]}$ via (7) with $\tau^{[s]}$; Update $\beta^{[s+1]}$ via $\left(\left[\right.\right.$ S.5) with $\tau^{[s]}, \rho^{[s]}$; Update $\alpha^{[s+1]}$ either via Equation (8) (histogram method) or (9) (kernel method) using the process $\tilde{N}^{(q, l)}$ and variational parameters $\rho^{[s]}$ and $\tau^{[s]}$.

- VE-step: Update the values $\rho^{[s+1]}$ via $(\mathrm{S} .3)$ with $\beta^{[s+1]}$ and $A^{[s+1]}(T)$ derived from $\alpha^{[s+1]}$; Update $\tau^{[s+1]}$ as the solution to the fixed point equation (S.4) relying on the current values $\pi^{[s+1]}, \rho^{[s+1]}, \alpha^{[s+1]}, \beta^{[s+1]}$. 
The integrated classification likelihood criterion becomes

$$
\operatorname{ICL}_{\text {sparse }}(Q)=\log \mathbb{P}_{\hat{\theta}(Q)}\{\mathcal{O}, \hat{\tau}(Q), \hat{\rho}(Q)\}-\frac{1}{2}(Q-1) \log n-\frac{1}{2} \log r\left[Q^{2}+\sum_{q=1}^{Q} \sum_{l=1}^{Q} 2^{\hat{d}^{(q, l)}}\right] .
$$

\section{S.7 The sparse setup: examples}

We first discuss the results of the sparse analysis on the London bike sharing system dataset. First let us recall that in this dataset only $7 \%$ of pairs of bike stations have at least one interaction. The main model ignores that fact and this impacts the results in the sense that groups are mainly driven by these absences of interactions. For instance the clusters obtained are mostly geographic, revealing absences of connections between distant bikes stations (nonetheless we also discovered an interesting small cluster with this model). We thus decide to explore whether one can decipher different structure with our sparse setup. In the following we focus on day 1 as similar results were obtained for day 2.

First our sparse integrated classification likelihood criterion selects only $\hat{Q}=2$ groups (compared to $\hat{Q}=6$ in the non sparse case). Geographic locations of the bike stations and the resulting clusters are represented on a city map (thanks to the OpenStreetMap project), see Figure S.12. There is one group containing a central part of the city while the remaining stations form a large peripheral cluster. Looking at the estimated intensities (Figure S.13) we see that the second group (i.e. the central geographical group) has large intra-group intensity with three modes: one in the morning (around 8:30 am), one at lunch (around 1pm) and the last at the end of the day (at 5:50pm). Group 1 (the peripheral one) mostly consists in 'leaving' stations in the morning (see mode in the estimated intensity for $(q, l)=(1,2)$ around 8:20am) and in 'arriving' stations at the end of the day (mode in the estimated intensity for $(q, l)=(2,1)$ at $5: 50 \mathrm{pm}$ ). Intra-group interactions in group 1 have a much lower intensity. On this dataset the sparse setup appears as a complementary model that may shed some different light on the data.

We also analysed the Enron corpus with the sparse model as $91 \%$ of the pairs of individuals do not exchange any email during the observation time. The sparse integrated classification likelihood criterion chooses $\hat{Q}=10$ as the optimal number of groups, which is smaller than in the non sparse model where no optimum has been found in the range of $Q$ from 1 to 20. As in the non sparse model the algorithm identifies one large group with 125 members, while the other nine groups contain at most five individuals. The adjusted rand index of the clustering in the sparse case with $Q=10$ (or $Q=4)$ and the clustering in the non sparse model with $Q=4$ equals $0.51(0.52)$, which means that there are substantial differences between the clusterings in the two models. Figure S.14 shows the estimated values of the connectivity probabilities $\beta_{q, l}$. Most of these probabilities are significantly lower than 1 justifying the application of the sparse model to these data. A consequence of low connectivity probabilities $\beta_{q, l}$ is that the estimated intensities are more elevated than in the non sparse case which can be observed in Figure $\mathrm{S} .15$ in comparison to the intensity values obtained in the non sparse model (Figure S.6. We can also compare the estimated intensities in the sparse model with $Q=4$ (Figure S.16 with those in the non sparse case. Again we see that the intensities in the sparse setup are much more elevated. Moreover, the form of the intensities involving two small groups (i.e. $\left.(q, l) \in\{2,3,4\}^{2}\right)$ are all quite different in the two models.

We conclude that as in the London bikes example the results in the sparse model differ much from those in the non sparse case. The sparse model tends to select a smaller number of groups 
which makes interpretation of results easier. As many real datasets are sparse in the sense that only a small percentage of individuals effectively interact with another the sparse model seems to be particularly adapted to real data and provides the possibility of further insights on the data.

We also analysed the primary school dataset with the sparse model. In this dataset $28 \%$ of pairs of individuals have at least one interaction. Our sparse integrated classification likelihood criterion selects $\hat{Q}=13$ groups, which is smaller than in the non sparse model. The clustering in the non sparse model and in the sparse model are quite close, with some groups being the same. The main difference between the two clusterings concerns the group composed in the non sparse model of class 4B with additional pupils coming from almost all other classes. This group was characterized by the lowest intra-group intensity. In the sparse model, class 4B is separated into two groups: 6 pupils are gathered with class 4A to form one group (group 1), whereas the 17 remaining pupils are gathered with class $1 \mathrm{~A}$ and some pupils coming from almost all other classes (group 3). Looking at the estimated intensities, we see that group 3 has a low intra-group intensity during the lunch time contrary to group 1 (see Figure S.17). Moreover the estimated intra-connectivity probability for groups 1 and 3 are given by $\hat{\beta}_{1,1}=0.84$ and $\hat{\beta}_{3,3}=0.29$. Therefore group 3 is composed of individuals which only a few proportion interacts, and characterized by very few interactions during the lunch time. On this dataset with the non sparse and sparse models we mainly recover the same clustering based on communities, but the sparse model also exhibits particular temporal profile of some individuals. 

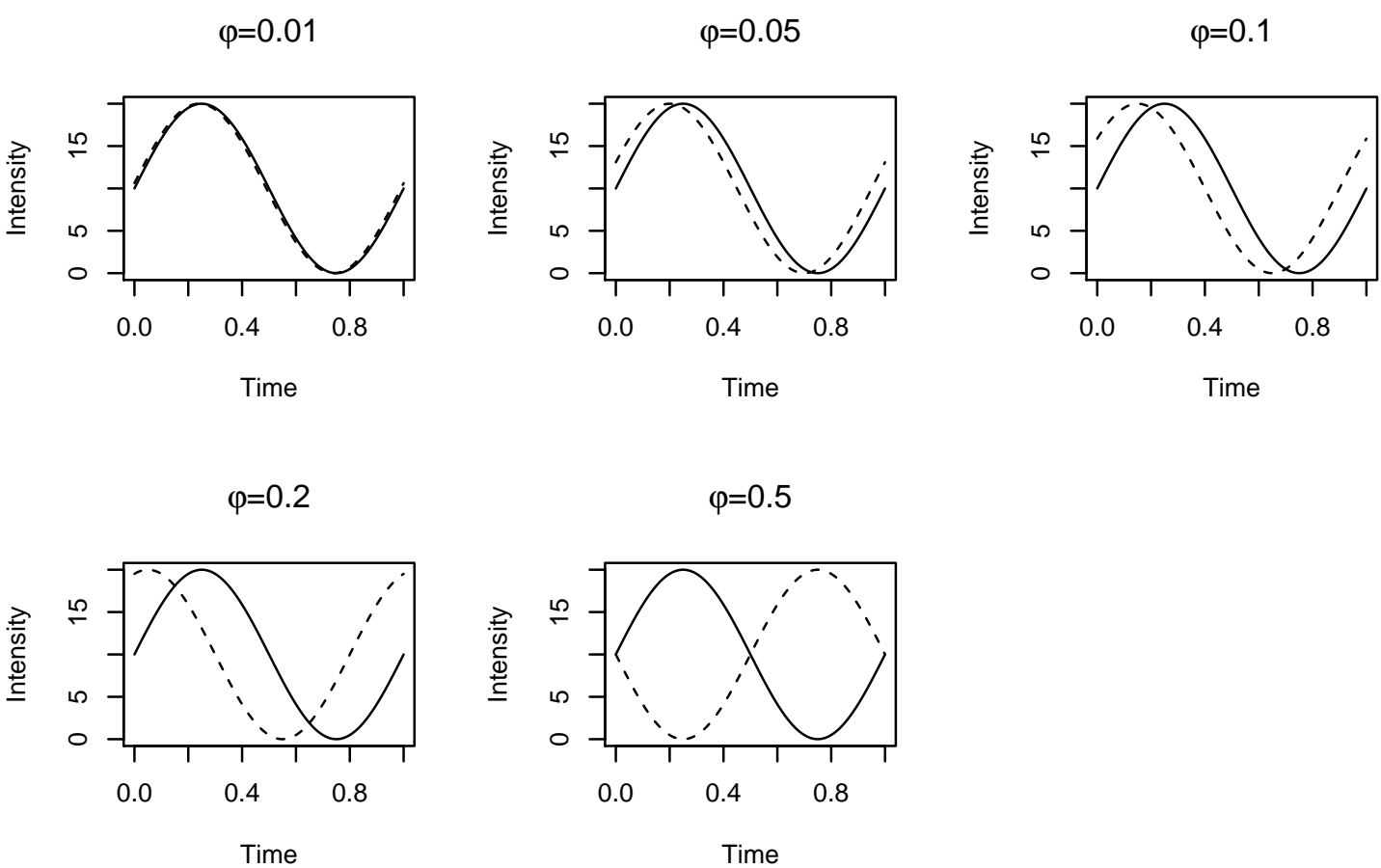

Figure S.1: Intensities in synthetic experiments from Scenario 1. Each picture represents the intra-group intensity $\alpha^{\text {in }}$ (bold line) and the inter-group intensity $\alpha^{\text {out }}$ (dotted line) with different shift parameter values $\varphi \in\{0.01,0.05,0.1,0.2,0.5\}$.

Table 2: Mean number of events and risks with standard deviations (sd) in scenario 2 with $n=20$. Histogram (Hist) and kernel (Ker) estimators are compared with their oracle counterparts (Or.Hist, Or.Ker). All values associated with the risks are multiplied by 100 .

Groups $(q, l) \quad$ Nb.events $\quad$ Hist (sd) Or.Hist (sd) $\quad$ Ker (sd) $\quad$ Or.Ker (sd)

$\begin{array}{cccccc}(1,1) & 84 & 136(92) & 50(49) & 215(83) & 113(55) \\ (1,2) & 146 & 177(146) & 98(27) & 270(107) & 194(23) \\ (1,3) & 86 & 211(160) & 78(20) & 178(143) & 43(18) \\ (2,2) & 32 & 136(72) & 108(29) & 139(109) & 71(41) \\ (2,3) & 130 & 265(72) & 217(28) & 238(78) & 182(22) \\ (3,3) & 48 & 173(61) & 158(47) & 171(111) & 85(43)\end{array}$




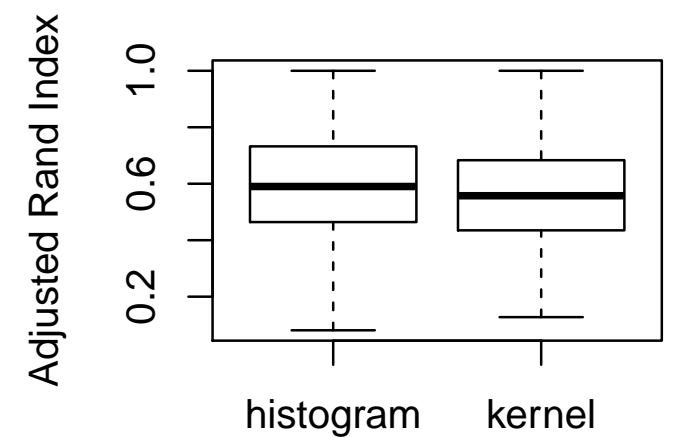

$\mathrm{n}=20$

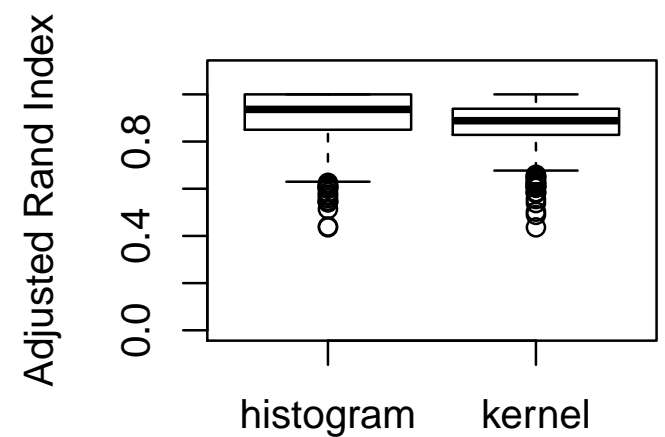

$\mathrm{n}=50$

Figure S.2: Boxplots of the adjusted rand index in synthetic experiments from Scenario 2, for the histogram (left) and the kernel (right) estimators. Left panel: $n=20$, right panel: $n=50$.

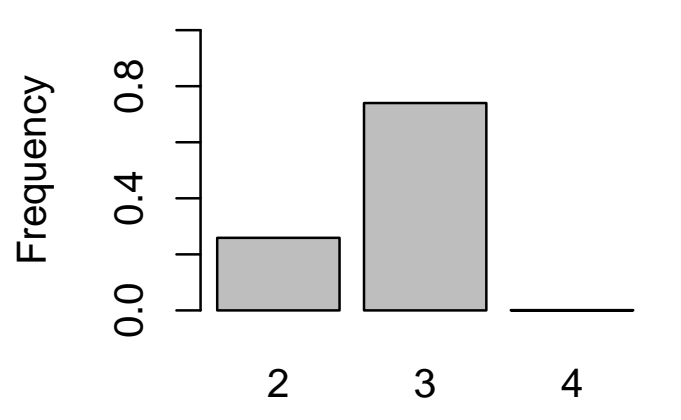

Selected number of groups

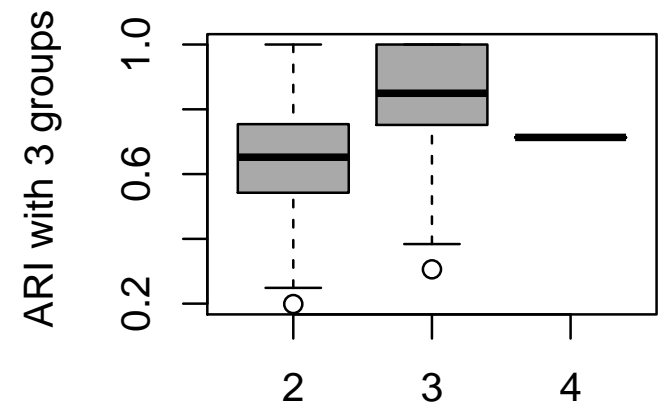

Selected number of groups

Figure S.3: Selection of the number of latent groups via the integrated classification likelihood criterion in Scenario 2 with $n=20$. Left panel: frequencies of selected number of groups. Right panel: adjusted rand index between the classification into three groups and true classification as a function of the number of selected groups by ICL(in $\{2,3,4\}$ ). 

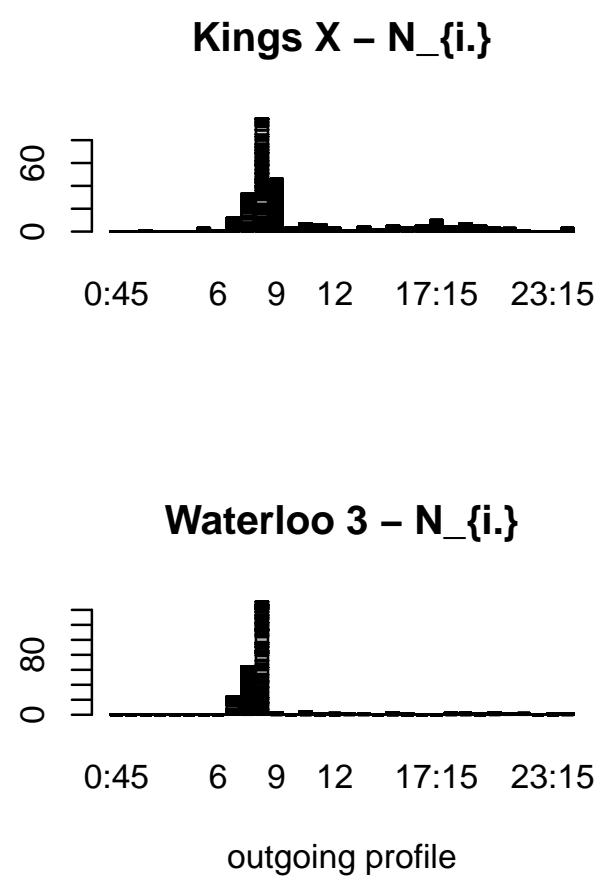
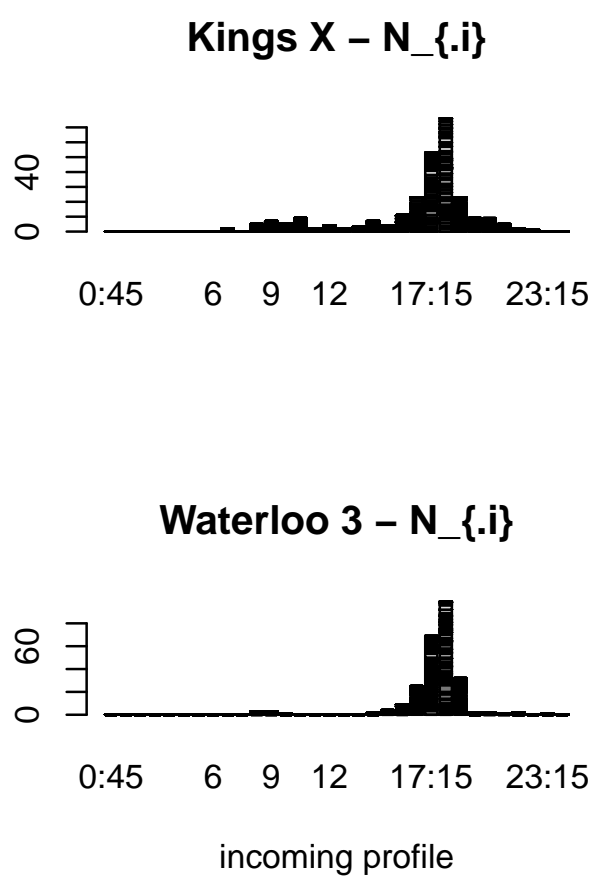

Figure S.4: London bike sharing system: Barplots of outgoing $\left(N_{i} \cdot(\cdot)\right.$ on the left) and incoming $\left(N_{\cdot i}(\cdot)\right.$ on the right) processes from the 2 stations $i$ (top row and bottom row, respectively) in the small cluster: representation of volumes of connections to all other stations during day 1 (time on the $x$-axis).

Table 3: Enron: Total size and group composition with $Q=4$ groups (some people's positions are unknown).

$$
\text { total managers employees }
$$

$\begin{array}{lccc}\text { group 1 } & 127 & 62 & 36 \\ \text { group 2 } & 4 & 0 & 3 \\ \text { group 3 } & 2 & 1 & 1 \\ \text { group 4 } & 14 & 12 & 1\end{array}$




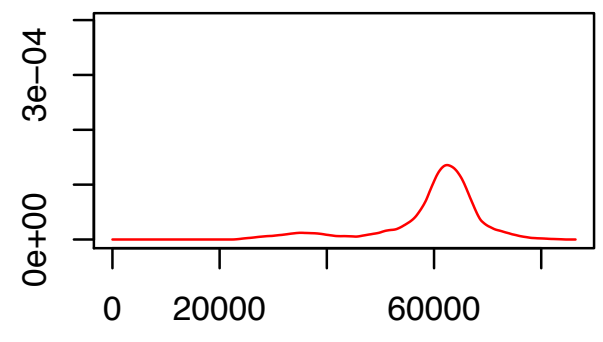

$(3,4)$

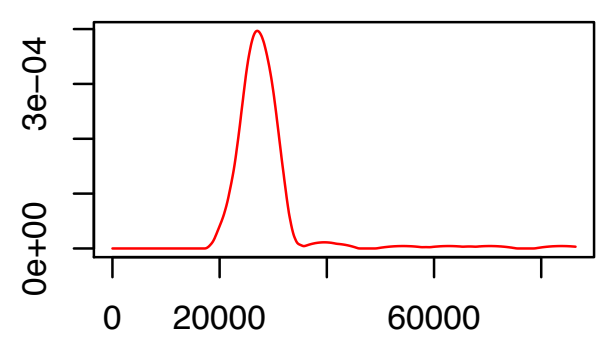

$(4,5)$

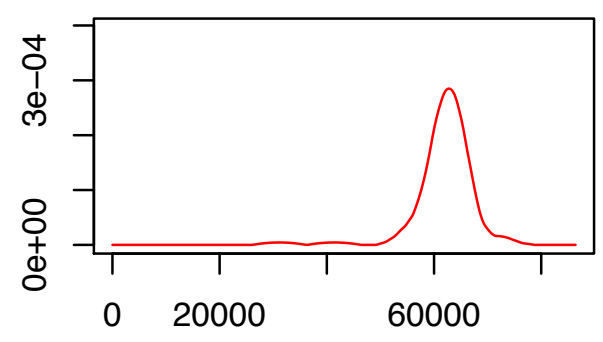

$(5,4)$

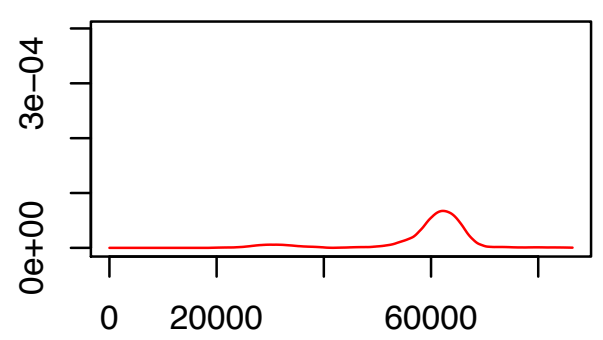

$(6,4)$

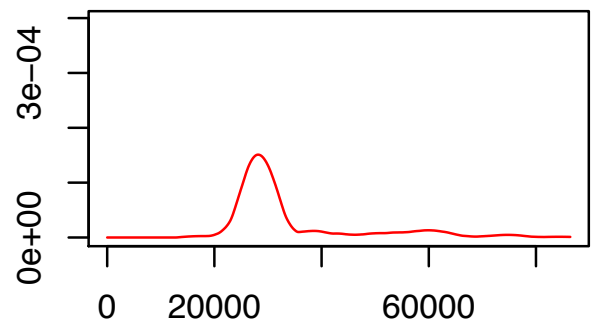

$(4,3)$

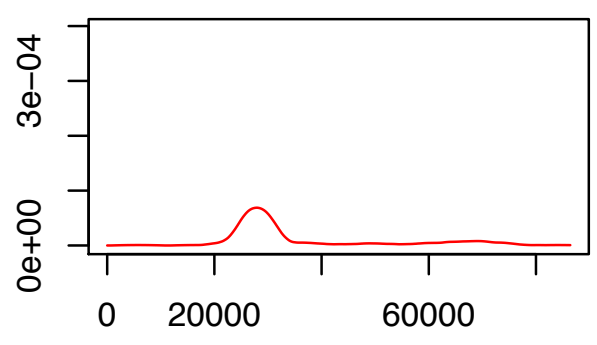

$(4,6)$

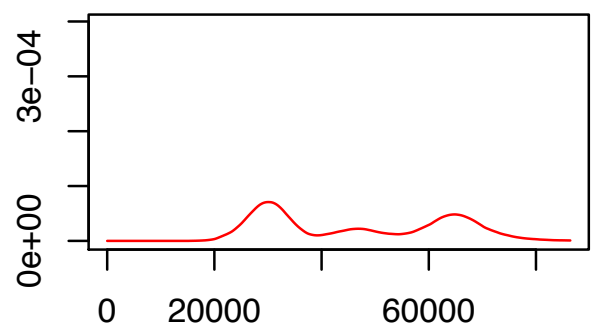

$(5,5)$

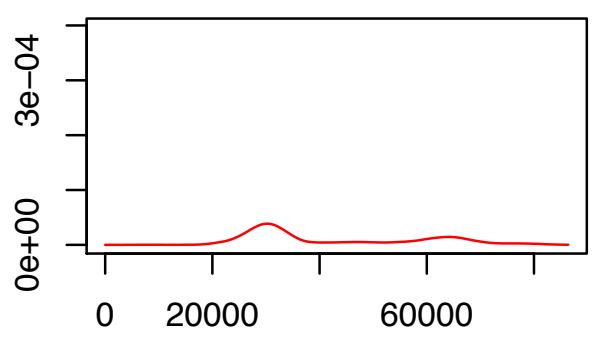

$(6,5)$

Figure S.5: London bike sharing system: estimated non almost null intensities for day 1 (time on the $x$-axis is in seconds). 


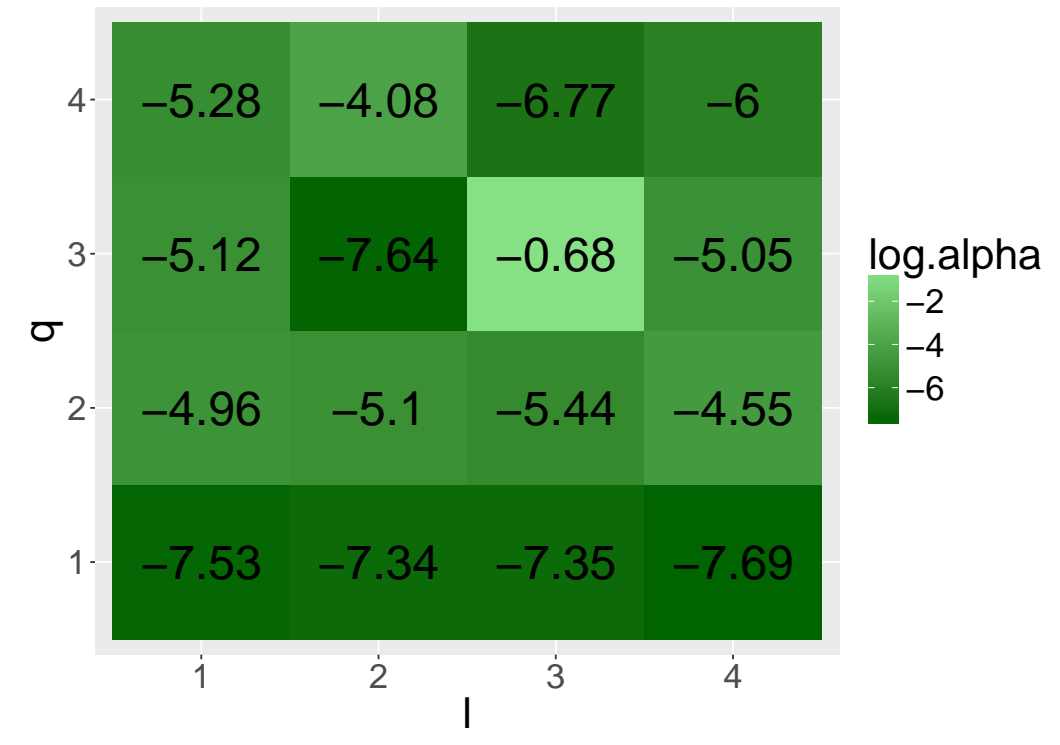

Figure S.6: Enron: Logarithm of the mean values of the estimated intensities $\alpha^{(q, l)}$ with $Q=4$ groups. 


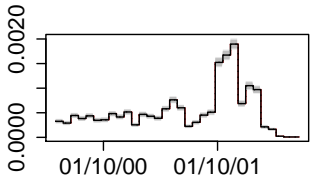

$(1,1)$

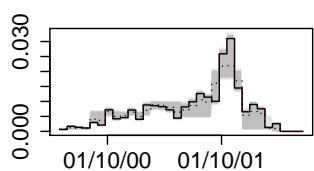

$(2,1)$

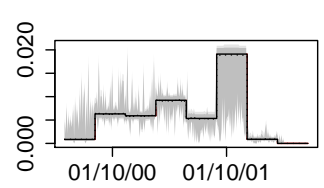

$(3,1)$

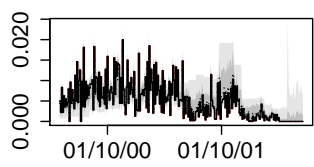

$(4,1)$

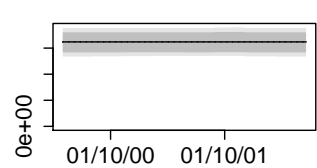

$(1,2)$

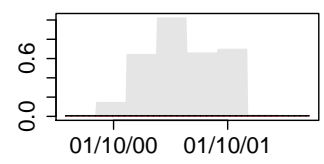

$(2,2)$

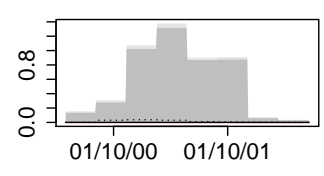

$(3,2)$

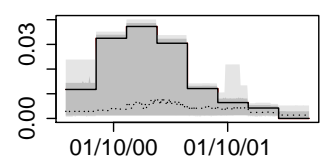

$4,2)$

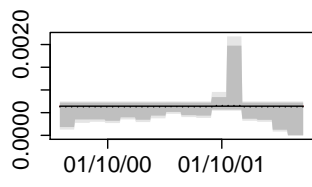

$(1,3)$

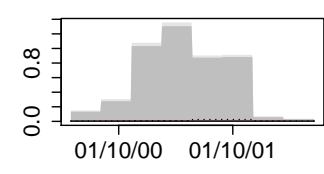

$(2,3)$

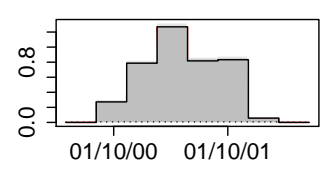

$(3,3)$

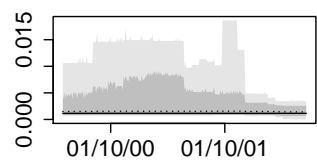

$(4,3)$
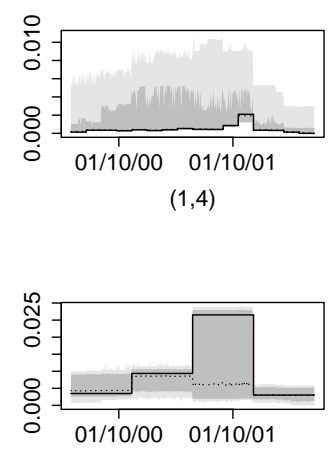

$(2,4)$
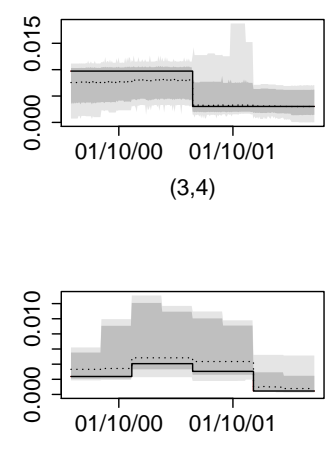

$(4,4)$

Figure S.7: Enron: Estimated intensities $\hat{\alpha}^{(q, l)}$ with $Q=4$ groups with bootstrap confidence intervals with confidence level 90\% (lightgrey) and 80\% (drakgrey) and the median bootstrap values (dotted lines). 


\section{$Q=17$}

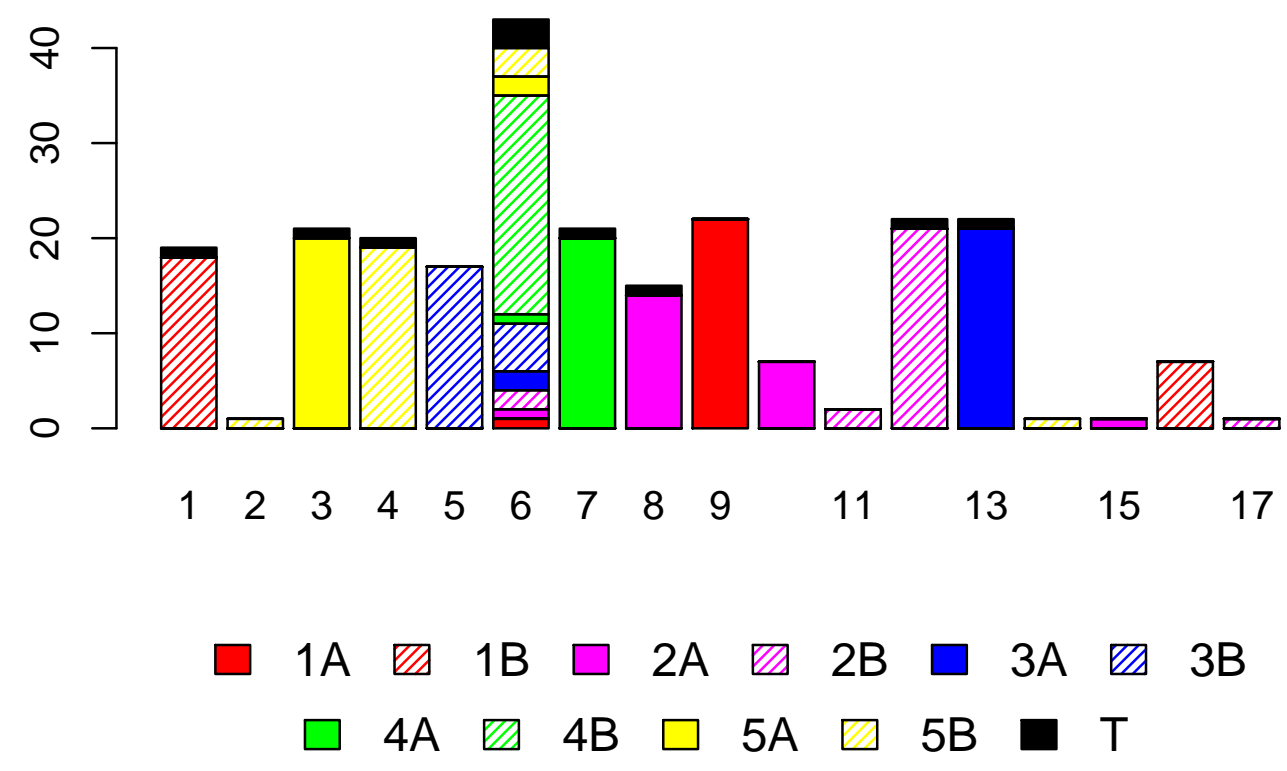

Figure S.8: Primary school: clustering of the 242 individuals into $Q=17$ groups. Vertical bars represent the $Q$ clusters. Colours indicate the grades and the teachers, plain and hatching distinguish the two classes in the same grade. 


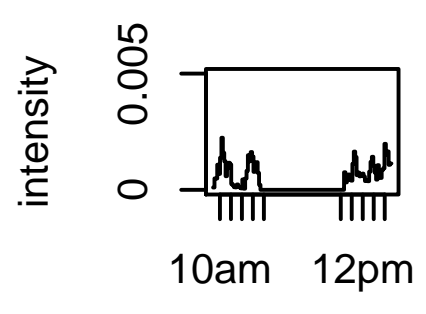

$(1,1)$

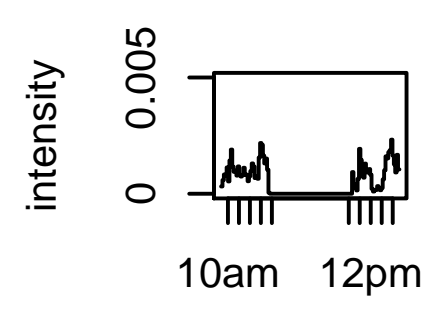

$(5,5)$

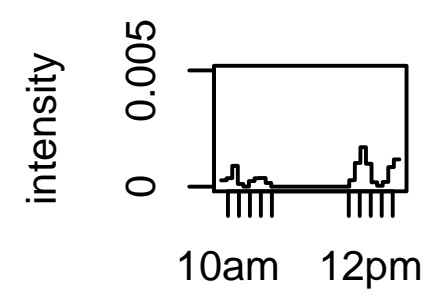

$(8,8)$

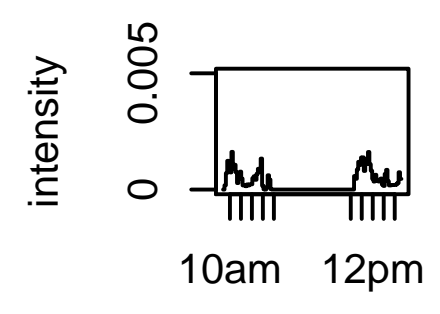

$(12,12)$

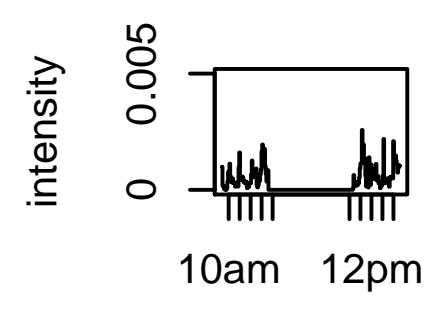

$(3,3)$

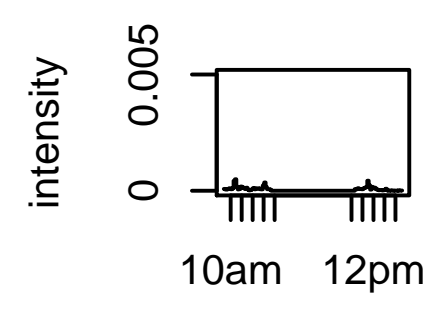

$(6,6)$

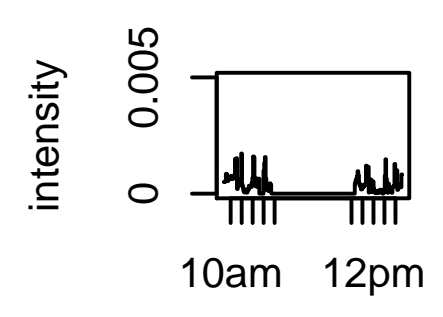

$(9,9)$

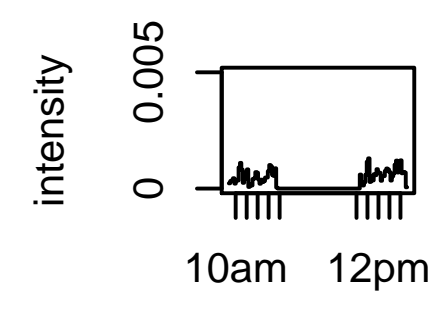

$\$ 83,13)$

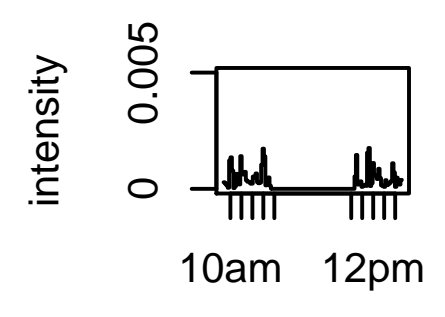

$(4,4)$

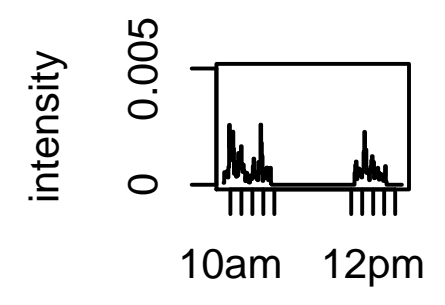

$(7,7)$
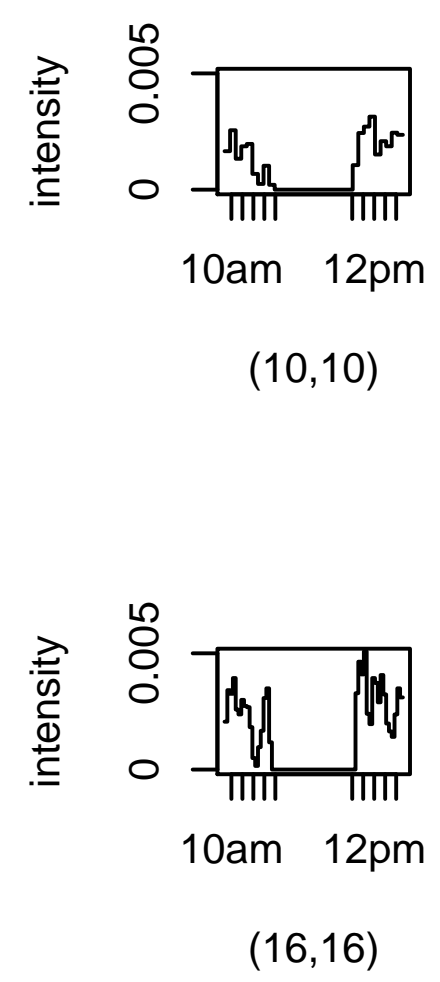

Figure S.9: Primary school: Estimated intra-group intensities (plotted on the same $y$-scale). 


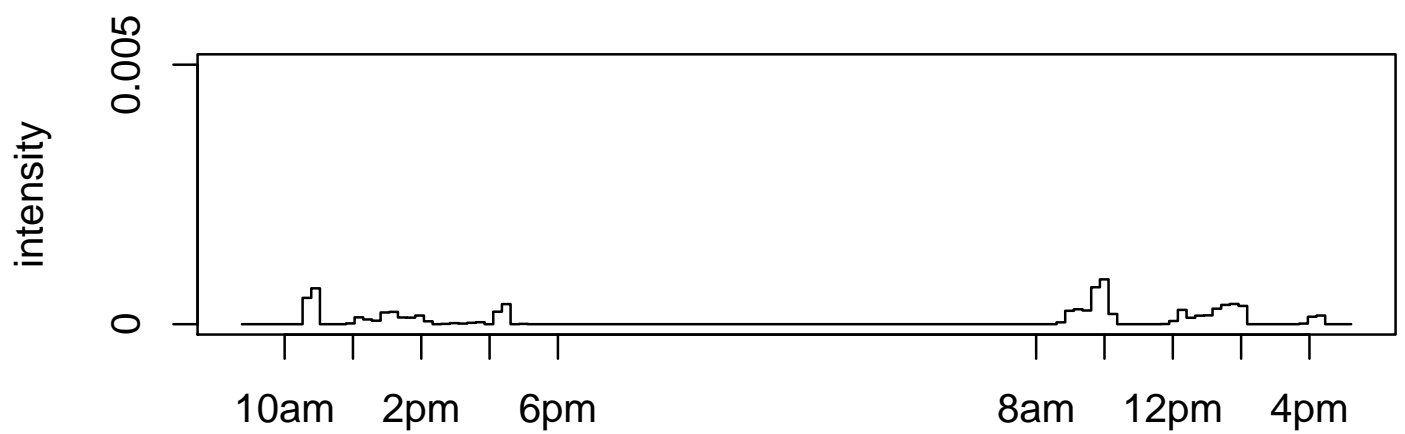

$(5,13)$

Figure S.10: Primary school: Estimated inter-group intensity between two classes of the same grade: classes $3 A$ (group 13) and $3 B$ (group 5).

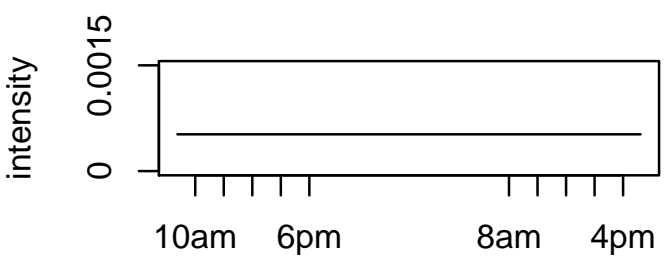

$(11,11)$

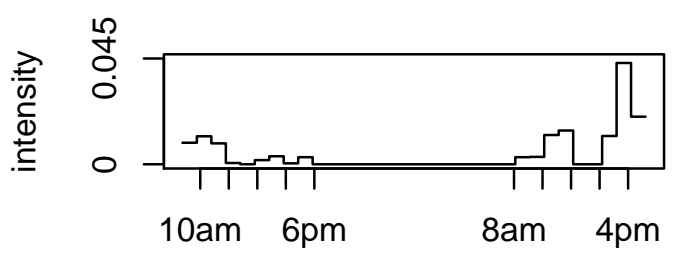

$(11,17)$

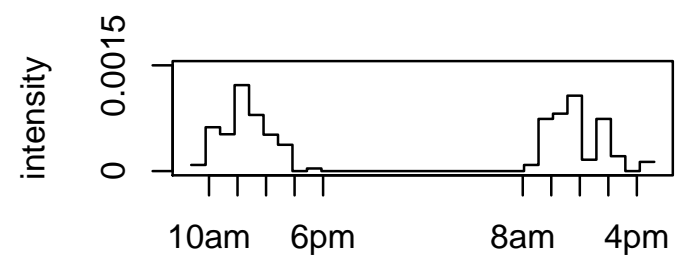

$(11,12)$

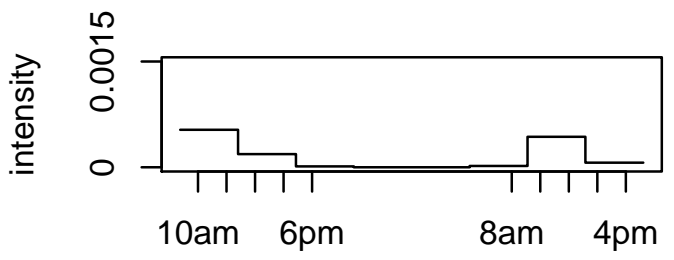

$(12,17)$

Figure S.11: Primary school: Estimated intensities. Example of class 2B splited into group 12 (with 21 pupils), group 11 (with 2 pupils), and group 17 (with only one pupil). 


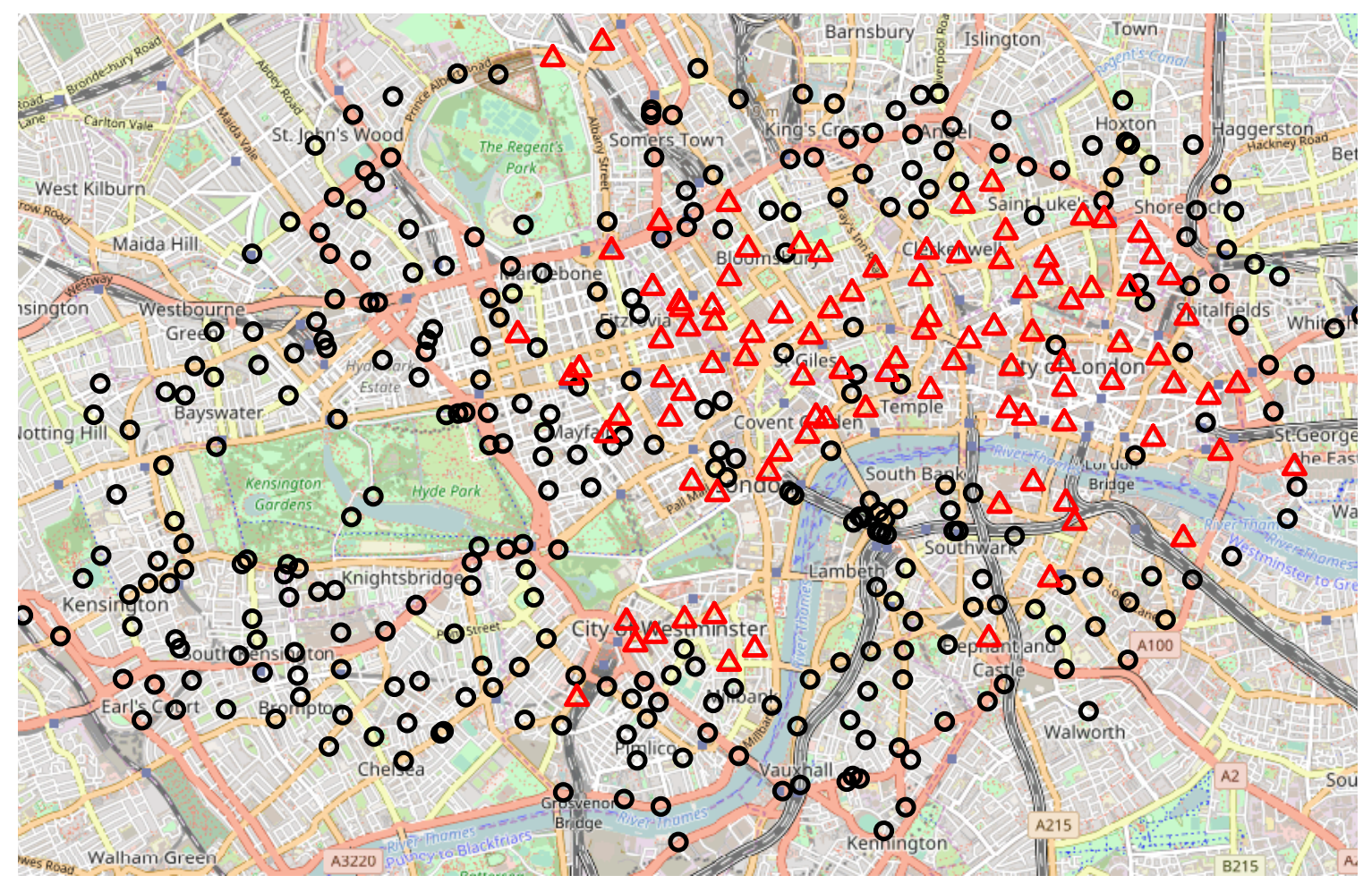

Figure S.12: London bike sharing system: Geographic positions of the stations and clustering into two clusters (represented by different colors and symbols) obtained from the sparse model for day 1 . 


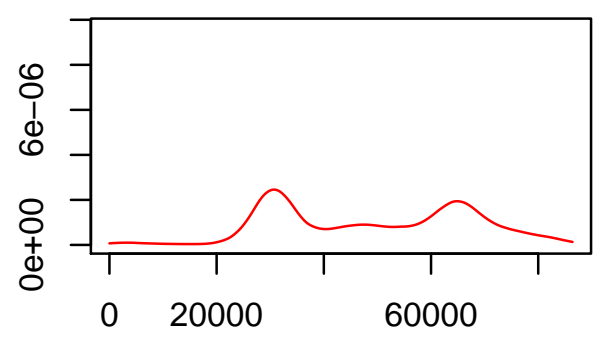

$(1,1)$

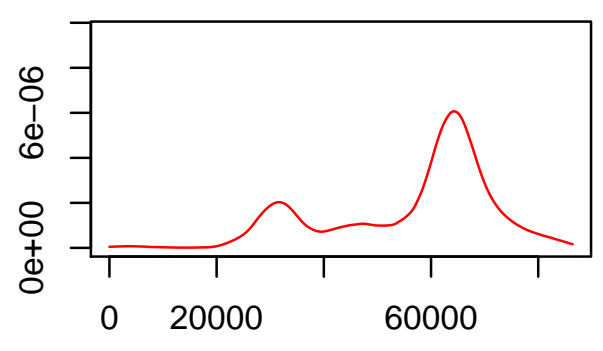

$(2,1)$

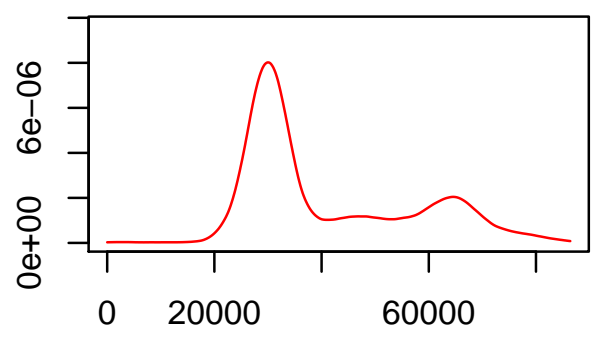

$(1,2)$

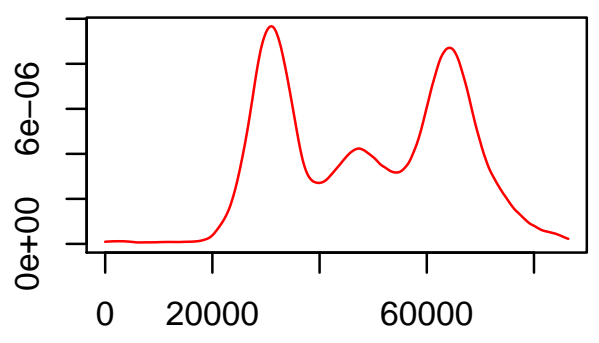

$(2,2)$

Figure S.13: London bike sharing system: estimated intensities from the sparse model for day 1 (time on the $x$-axis is in seconds). 


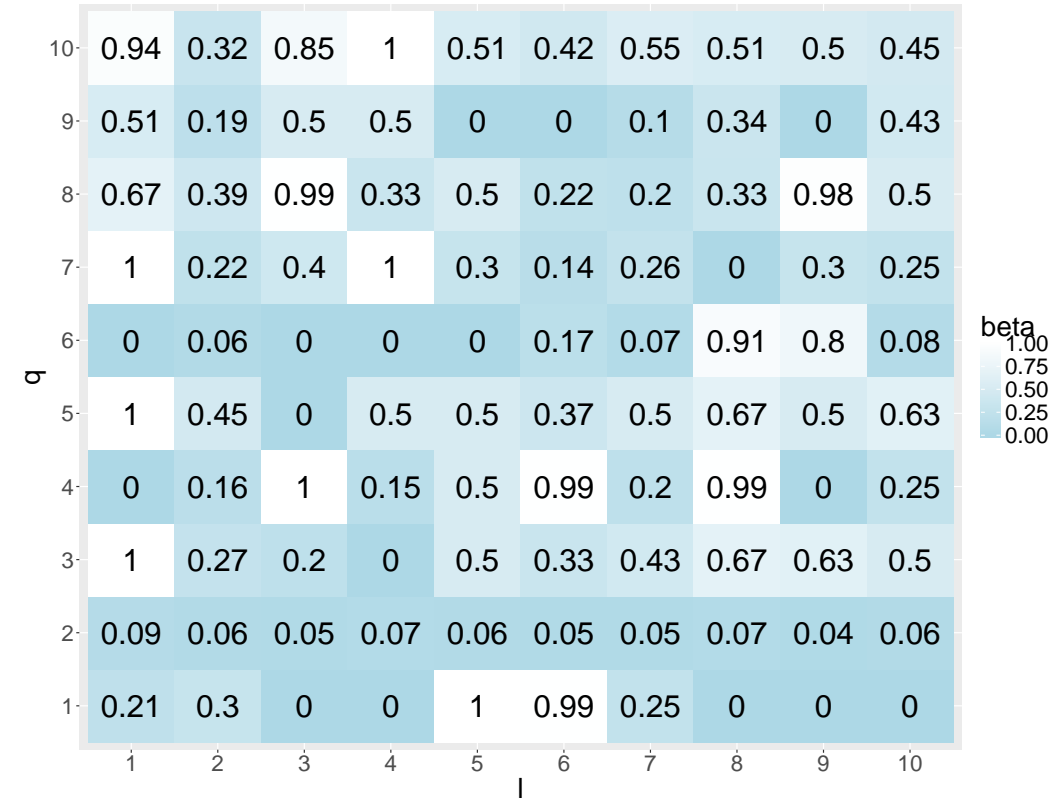

Figure S.14: Enron: Estimated connectivity probabilities $\beta_{q, l}$ in the sparse model with $Q=10$ groups.

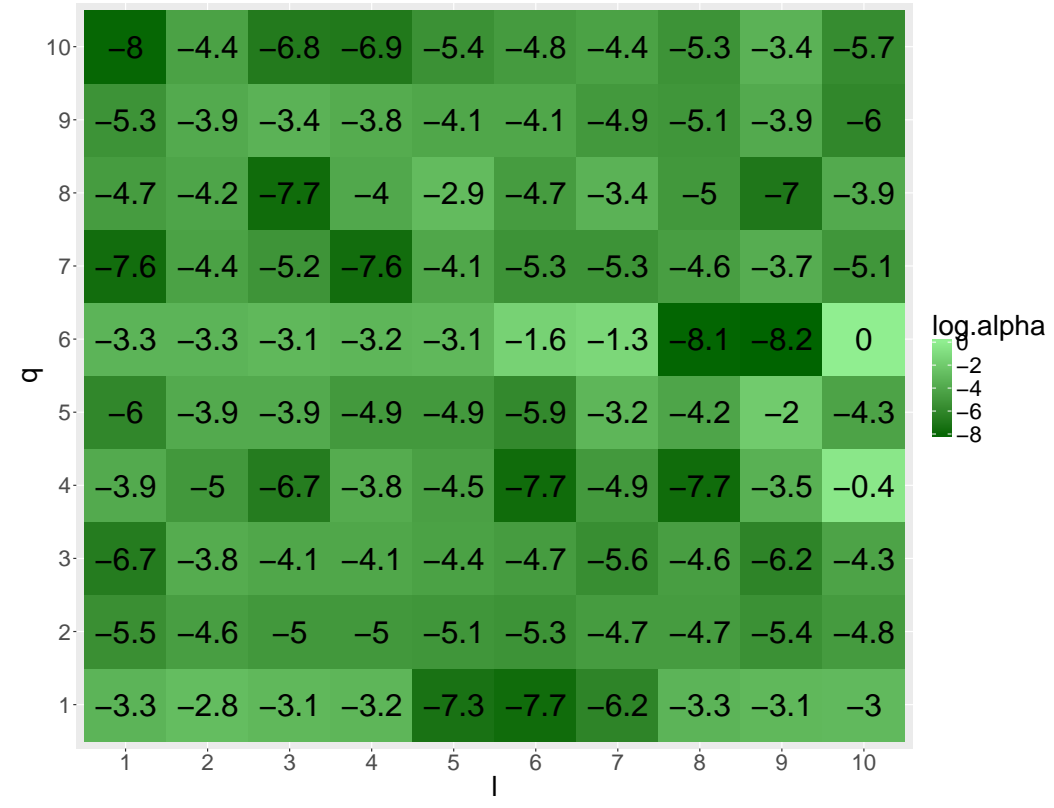

Figure S.15: Enron: Logarithm of the mean values of the estimated intensities $\alpha^{(q, l)}$ in the sparse model with $Q=10$ groups. 

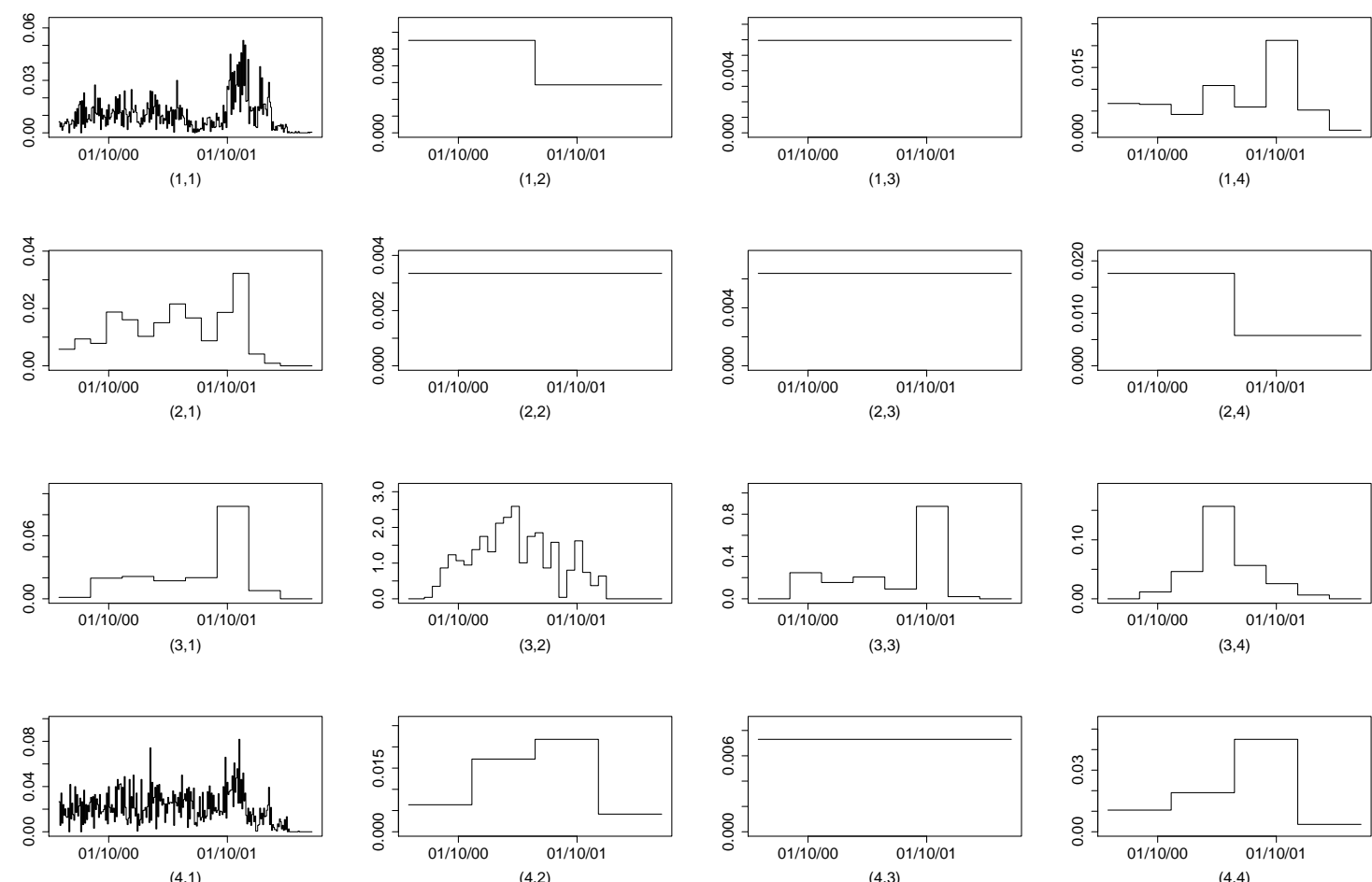

Figure S.16: Enron: Estimated intensities $\hat{\alpha}^{(q, l)}$ in the sparse model with $Q=4$ groups. 


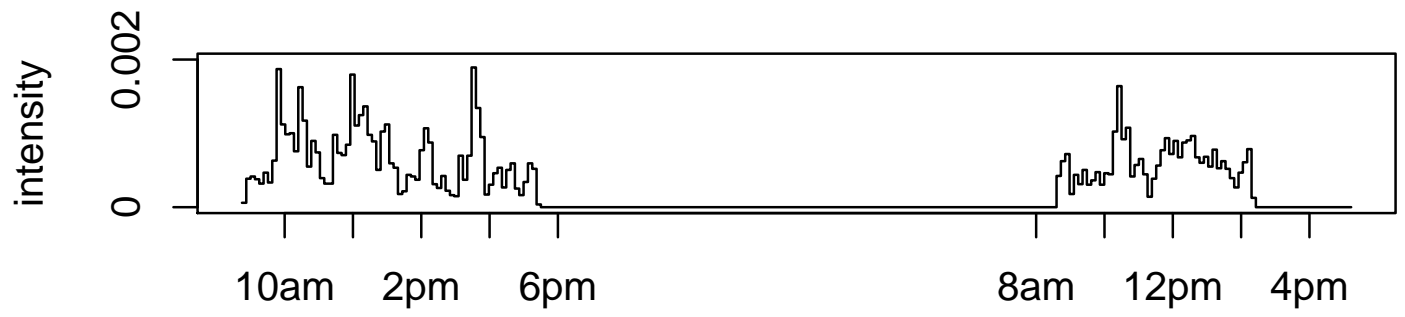

$(1,1)$

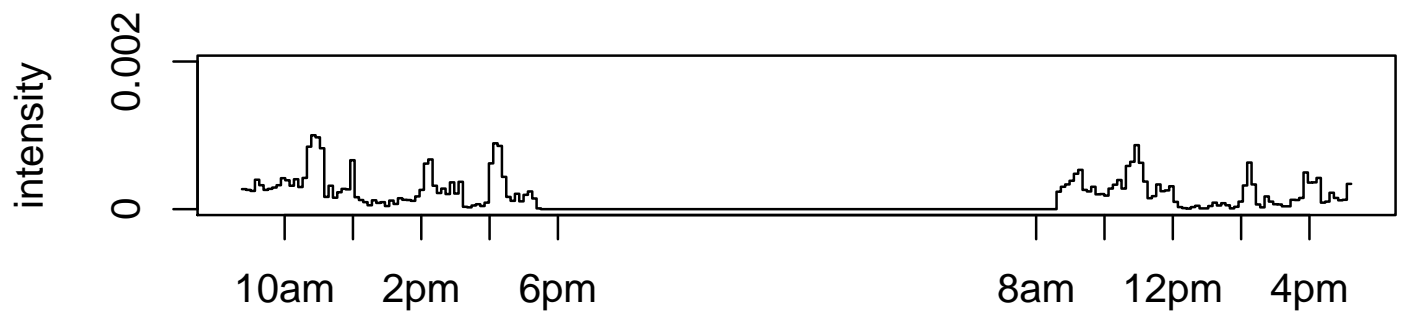

$(3,3)$

Figure S.17: Primary school: Estimated intensities in the sparse model. Example of group 1 (composed of class 4A and 6 pupils of class 4B) and group 3 (composed of the entire class $1 \mathrm{~A}$, with 17 pupils of class $4 \mathrm{~B}$ and pupils from almost all other classes).

\section{References}

E. Allman, C. Matias, and J. Rhodes. Parameters identifiability in a class of random graph mixture models. J. Stat. Plan. Inference, 141:1719-1736, 2011.

D. J. Daley and D. Vere-Jones. An introduction to the theory of point processes. Vol. I. Probability and its Applications (New York). Springer-Verlag, New York, second edition, 2003. Elementary theory and methods.

SocioPatterns. http://www.sociopatterns.org/, 2015.

J. Stehlé, N. Voirin, A. Barrat, C. Cattuto, L. Isella, J.-F. Pinton, and et al. High-resolution measurements of face-to-face contact patterns in a primary school. PLoS ONE, 6(8):e23176, 2011. 